\title{
Whole Earth telescope observations of the ZZ Ceti star HL Tau 76 $6^{\star \star \star \star}$
}

\author{
N. Dolez ${ }^{1}$, G. Vauclair ${ }^{1}$, S. J. Kleinman ${ }^{2}$, M. Chevreton ${ }^{3}$, J. N. Fu ${ }^{1,20}$, J.-E. Solheim ${ }^{4,21}$, J. M. González Perez ${ }^{4,22}$, \\ A. Ulla ${ }^{5}$, L. Fraga ${ }^{6}$, A. Kanaan ${ }^{6}$, M. Reed ${ }^{7,23}$, S. Kawaler ${ }^{7}$, M. S. O'Brien ${ }^{7}$, T. S. Metcalfe ${ }^{8,24}$, R. E. Nather ${ }^{8}$, \\ D. Sanwal $^{8}$, E. W. Klumpe ${ }^{8,25}$, A. Mukadam ${ }^{8}$, M. A. Wood ${ }^{9}$, T. J. Ahrens ${ }^{9}$, N. Silvestri ${ }^{9,26}$, D. Sullivan ${ }^{10}$, \\ T. Sullivan ${ }^{10}$, X. J. Jiang ${ }^{11}$, D. W. Xu ${ }^{11}$, B. N. Ashoka ${ }^{12}$, E. Leibowitz ${ }^{13}$, P. Ibbetson ${ }^{13}$, E. Ofek ${ }^{13}$, D. Kilkenny ${ }^{14}$, \\ E. G. Meištas ${ }^{15}$, D. Alisauskas ${ }^{15}$, R. Janulis ${ }^{15}$, R. Kalytis ${ }^{15}$, P. Moskalik ${ }^{16}$, S. Zola ${ }^{17}$, J. Krzesinski ${ }^{17}$, W. Ogloza ${ }^{17}$, \\ G. Handler ${ }^{18,14}$, R. Silvotti ${ }^{19}$, and S. Bernabei ${ }^{19}$ \\ (Affiliations can be found after the references)
}

Received 29 March 2005 / Accepted 12 September 2005

\begin{abstract}
This paper analyses the Whole Earth Telescope observations of HL Tau 76, the first discovered pulsating DA white dwarf. The star was observed during two Whole Earth Telescope campaigns. It was a second priority target during the XCOV13 campaign in 1996 and the first priority one during the XCOV18 campaign in 1999. The 1999 campaign reached $66.5 \%$ duty cycle. With a total duration of 18 days, the frequency resolution achieved is $0.68 \mu \mathrm{Hz}$. With such a frequency resolution, we were able to find as many as 78 significant frequencies in the power spectrum, of which 34 are independent frequencies after removal of all linear combinations. In taking into account other frequencies present during the 1996 WET campaign and those present in earlier data, which do not show up in the 1999 data set, we find a total of 43 independent frequencies. This makes HL Tau 76 the richest ZZ Ceti star in terms of number of observed pulsation modes. We use those pulsation frequencies to determine as much as possible of the internal structure of HL Tau 76. The pulsations in HL Tau 76 cover a wide range of periods between $380 \mathrm{~s}$ and $1390 \mathrm{~s}$. We propose an identification for 39 of those 43 frequencies in terms of $\ell=1$ and $\ell=2$ non-radial $g$-modes split by rotation. We derive an average rotation period of 2.2 days. The period distribution of HL Tau 76 is best reproduced if the star has a moderately "thick" hydrogen mass fraction $\log q_{\mathrm{H}} \geq-7.0$. The results presented in this paper constitute a starting point for a detailed comparison of the observed periods with the periods calculated for models as representative as possible of HL Tau 76.
\end{abstract}

Key words. stars: interiors - stars: individual: HL Tau 76 - stars: oscillations - stars: white dwarfs

\section{Introduction}

There are 71 pulsating DA white dwarfs presently known (the $\mathrm{ZZ}$ Ceti or DAV stars). This number is composed on one hand of the $36 \mathrm{ZZ}$ Ceti stars published prior to the first release of the Sloan Digital Sky Survey (SDSS) and includes the ZZ Ceti stars recently discovered on the basis of their location in the $\log g-T_{\text {eff }}$ diagram: G30-20 (Mukadam et al. 2002), MCT 0145-2211 and HE 0532-5605 (Fontaine et al. 2003),

^ Based on data obtained at the Haute-Provence Observatory, INSU/CNRS, France, the Teide Observatory, Tenerife, Spain, the Laboratorio Nacional de Astrofisica/CNPq, Brazil, the Cerro Tololo Interamericain Observatory, Chili, the McDonald Observatory, Texas, USA, the Kitt Peak National Observatory, Arizona, USA, the Mauna Kea Observatory, University of Hawaii, USA, the Beijing Observatory, China, the Naini Tal Observatory, India, The South Africa Astronomical Observatory, South Africa, the Moletai Observatory, Lituania, the Wise Observatory, Israel, the Suhora Observatory, Poland and the Loiano Observatory, Italy.

$\star \star$ Table 2 is only available in electronic form at http://www.edpsciences.org
LP133-144 and HE1258+0123 (Bergeron et al. 2004). They lie well inside the ZZ Ceti instability strip as defined by Bergeron et al. (1995). On the other hand, 35 new ZZ Ceti stars have been discovered from the first release catalog of the SDSS (Mukadam et al. 2004a; Abazajian et al. 2003; Harris et al. 2003; Kleinman et al. 2004). The 36 previously published ZZ Ceti stars define a narrow instability strip in the H-R diagram (or in the $\log g-T_{\text {eff diagram) of trapezoidal form due }}$ to the mass dependence of its blue and red edges. Its location in $T_{\text {eff }}$ depends on the adopted prescription for the convection. Bergeron et al. (1995) have shown that within the framework of the mixing lenght theory (MLT), the choice of the ML2 version with a mixing length equals to 0.6 pressure height scale optimizes the Balmer lines profiles and the energy distribution from the UV to the visible wavelength range. For the average DA mass, it ranges from $12460 \pm 200 \mathrm{~K}$ for the hottest (G226-29) to $11070 \pm 200 \mathrm{~K}$ for the coolest (G30-20 and BPM24754), according to Bergeron et al. (1995), Fontaine et al. (2003) and Bergeron et al. (2004). According to Bergeron et al. (2004), the instability strip for these ZZ Ceti 
white dwarfs is a "true" instability strip, which means that all the DA white dwarfs falling inside this region of the H-R diagram do pulsate (no non-pulsating DA has yet been found within the instability strip in this sample). This is in disagreement with the instability strip derived from the SDSS newly discovered ZZ Ceti stars. Mukadam et al. (2004b) suggest that it is not pure. Whether this results from a genuine cohabitation of pulsators and non-pulsators within the instability strip or from larger uncertainties in the atmospheric parameters derived from the SDSS needs to be examined. The DA white dwarfs of the SDSS sample falling within the instability strip are fainter than those analyzed by Bergeron et al. (2004). Their atmospheric parameters are derived from lower $S / N$ spectra: for instance, the $S / N$ of the spectrum for the brighest non variable star (WD 1338-0023, $g=17.1$ ) in the Mukadam et al. (2004b) sample is 35 (Kleinman et al. 2004), while all the DA spectra analyzed by Bergeron et al. (2004) have a $S / N \geq 80$. It suggests that the location of the SDSS DA white dwarfs in the $\log g-T_{\text {eff }}$ diagram suffers larger uncertainties. This may explain the overlap between pulsators and non-pulsators within the Mukadam et al. (2004b) instability strip.

Whether the instability strip is pure or not pure is an important issue since, if the instability strip is a "pure" one, then it is an indication that the internal structure of the ZZ Ceti white dwarfs as derived from asteroseismology is representative of the DA white dwarfs as a whole group. DA white dwarfs represent $80 \%$ of the total white dwarf population (Fleming et al. 1986). As the white dwarfs are the last evolutionary stage of low and intermediate mass stars, which form the vast majority of all stars, it is important to know their internal structure as precisely as possible.

The white dwarf cooling sequence could potentially be used as a powerful cosmochronological tool (Winget et al. 1987). One finds most of the known white dwarfs in the solar neighbourhood, but they are also present in both open and globular clusters, and some have been identified as potential galactic halo members. In each case, calibrating the corresponding cooling sequence would provide an age determination, independent from other methods, and hopefully more precise. This goal is not yet achieved however, due to various uncertainties in the models. Among those uncertainties, the unknown proportion and distribution of $\mathrm{C}$ and $\mathrm{O}$ in the core and the incomplete understanding of the way they crystallize at the low luminosity end of the cooling sequence affect the estimate of the total thermal energy reservoir, while the unknown helium and hydrogen content and thickness of the envelope affects the estimate of the rate at which this energy is released through radiation and/or convection. They introduce an uncertainty on the derived age of the coolest white dwarfs on the order of 2 Gyr (Winget \& Van Horn 1987; see Fontaine et al. 2001 for a recent review).

The asteroseismological study of the ZZ Ceti white dwarfs can help in reducing part of these uncertainties by constraining the stars' total mass and hydrogen mass fraction. The difficulty in determining these values from asteroseismology comes from two main sources: 1) they show generally few modes, in contrast with the theoretical calculations which predict many more unstable modes than observed; and 2) the pulsation amplitudes become increasingly variable as the ZZ Ceti stars become closer to the red edge of the instability strip so that some of the modes may have amplitude below the detection limit during a given observing season, which implies that more than one observing season is necessary to recover a useful power spectrum. Both effects make it difficult to find enough modes to apply the standard method of asteroseismology which needs a sufficient number of pulsation modes to be observed and identified.

Most of the ZZ Ceti stars close to the instability strip blue edge show constant pulsation amplitude. But in this case, very few modes are unstable since the stars are just entering the instability strip. In those stars, the $\kappa-\gamma$ mechanism due to hydrogen partial ionization is responsible for the instability since the fraction of the flux conveyed by convection is negligible (Dolez \& Vauclair 1981; Winget et al. 1982). However, as they show too few modes for traditional pattern matching analysis, one can only rely on a direct comparison of the frequencies computed in white dwarf models with the observed frequencies to constrain the model, or a set of plausible models, which best reproduce the observations. There is generally no unique solution. As the white dwarfs cool within the instability strip, convection carries an increasing fraction of the flux as $T_{\text {eff }}$ decreases. Brickhill (1983, 1990, 1991a,b) found that since the convection zone responds almost instantaneously to the pulsations, it can drive the pulsations. The convective driving in ZZ Ceti stars was further analyzed by Goldreich \& Wu (Wu \& Goldreich 1999, 2001; Goldreich \& Wu 1999a,b; Wu 2001). As the ZZ Ceti cool and the convection zone deepens, more and more modes are driven and the convection give them increasing energy (and so more power). Since the depth of the convection zone varies in response to the variations of the physical conditions induced by the pulsations (temperature and pressure) non linearities occur in the surface flux perturbations which show up through an increasing complexity of the power spectra, variability of the amplitudes, non-sinusoidal pulse shapes, linear combinations of frequencies, etc. Closer to the red edge, almost all the flux is carried by convection. The strong feedback of convection on the pulsations now results in the damping of many of the modes, so that again fewer modes are available for asteroseismological diagnosis. So, the determination of the hydrogen mass fraction for individual ZZ Ceti stars has been attempted in very few cases. This led Clemens (1993) to consider the pulsation properties of the ZZ Ceti stars as a group. From the similarities in the period distribution observed in the hot ZZ Ceti stars, he concluded that they should also have a somehow similar internal structure and estimated that their hydrogen mass fraction should be around $M_{\mathrm{H}} / M_{*}=10^{-4}$, the value predicted by stellar evolution theory.

Looking for the ZZ Ceti stars for which a hydrogen mass determination has been proposed, one can check that in most cases those determinations rely on a small number of detected modes whose identification remains often ambiguous. Table 1 summarizes the results on the hydrogen mass fraction determined from asteroseismology. Except for G29-38, which is the best studied ZZ Ceti star up to now and in which 19 multiplets have been identified as possible $\ell=1$ modes, the number of available modes is small. In 5 of the ZZ Ceti stars listed, out of 8 total, the hydrogen mass fraction is estimated 
Table 1. Derived $M_{\mathrm{H}} / M_{\mathrm{WD}}$ in ZZ Ceti stars.

\begin{tabular}{lccc}
\hline \hline Object & Number of modes & $M_{\mathrm{H}} \times 10^{-4} M_{\mathrm{WD}}$ & Ref. \\
\hline G 226-29 & 3 & $\approx 1.0$ & 1 \\
L 19-2 & 11 & $\approx 1.0$ & 2 \\
R 548 & 7 & 1.5 & 3 \\
GD 165 & 8 & $\approx 2.0$ & 4 \\
G 29-38 & 19 & 0.005 & 5 \\
G 117-B15A & 3 & $\approx 1.0$ & 6 \\
GD 154 & 3 & $2 \times 10^{-6}$ & 7 \\
BPM 37093 & 8 & $0.02-0.6$ & 8 \\
\hline
\end{tabular}

Notes: The number of modes is obtained after subtracting the linear combinations and harmonics from the power spectra. Its does include multiplet components induced by rotational splitting (except in the case of G 29-38 where the indicated number is the total number of multiplets). This number is the sum of all the modes observed during various observing seasons. References: 1) Kepler et al. (1995); 2) Bradley (1993, 2001); 3) Bradley (1998); 4) Bergeron et al. (1993), Bradley (2001); 5) Kleinman et al. (1998); 6) Robinson et al. (1995), Bradley (1998); 7) Pfeiffer et al. (1996); 8) Kanaan et al. (2005), Metcalfe et al. (2004), but see also Fontaine \& Brassard (2004).

to be $\approx 10^{-4} M_{\mathrm{WD}}$, where $M_{\mathrm{WD}}$ is the white dwarf mass. These estimates rely mostly on the assumption that the shortest observed periods are correctly identified as the periods of the lowest order $\ell=1$ modes calculated in DA white dwarf models. In some of those models, the effects of varying the helium mass fraction has been included in the analysis, while in others models a standard value of $10^{-2}$ has been adopted as in the Wood's (1995) evolutionary sequences. In the other three cases, G29-38, GD154 and BPM37093, it is smaller. However, it must be emphasized that in the case of G29-38, there is not yet a convincing model so that the quoted value is only a preliminary estimate. In the case of GD 154, the estimate is based on the assumption that the three independent modes seen during the WET campaign were trapped modes, an assumption which may not be generally true since in the pulsating white dwarfs which show more modes, the largest amplitude ones are not necessarily trapped. For BPM37093, the hydrogen mass fraction is not strongly constrained since the possible models which fit equally well the observed periods cover a rather large range.

Since the hydrogen mass fraction estimate largely relies on a proper identification of the modes, which is often difficult even from long time-series photometric data, two alternative methods have been devised. In the first case, the wavelength dependence of the pulsation amplitudes has been used to select the $\ell$ degree of the modes (Robinson et al. 1995; Kepler et al. 2000b). The method requires data covering a large enough wavelength range, including preferably the UV where the amplitudes vary most significantly. It also requires high $\mathrm{S} / \mathrm{N}$ ratio to distinguish between $\ell=1$ and $\ell=2$ modes whose predicted amplitude variations are quite close. The second method uses time-dependent spectrophotometry (van Kerkwijk et al. 2000; Clemens et al. 2000; Kotak et al. 2002a; Thompson et al. $2003,2004)$. The variations in the line profile during the pulsation cycle contain information on the $\ell$ degree of the modes.
This method implies the use of large telescopes since it relies on high resolution spectroscopy with good enough $\mathrm{S} / \mathrm{N}$ on time scale which should be a fraction of the typical periods. However, both methods would also require long enough timeseries to resolve the close frequencies of rotationally split multiplets or of overlapping modes of different $\ell$ degrees, a condition which cannot be easily satisfied.

To summarize, the question of whether the DA white dwarfs all have the same hydrogen mass fraction is presently unsettled. A hydrogen mass fraction of $10^{-4} M_{\mathrm{WD}}$ is adopted as a "standard" value in most models used in computing cooling sequence (the so called "thick" hydrogen models). If a large dispersion in the hydrogen mass fraction in DA white dwarfs was found to be real, it would introduce an equivalent dispersion in the age of white dwarfs of a given luminosity and increase the uncertainty in the age calibration of the white dwarf luminosity function.

So, trying to determine the hydrogen mass fraction in DA white dwarfs from the asteroseismology of the ZZ Ceti stars is worthwhile. HL Tau 76 is a ZZ Ceti star of particular interest in this respect. It shows a large number of pulsation frequencies whose hopefully a significant fraction should be independent pulsation modes. As a result, the model is expected to be rather strongly constrained and its main parameters, including its hydrogen mass fraction, well determined.

The star was the primary target of a Whole Earth Telescope (WET, Nather et al. 1990) campaign whose results are described in the present paper. In Sect. 2, the observations and the data reduction are presented. Section 3 gives a global description of the power spectrum while Sect. 4 offers a detailed analysis of the power spectrum and a discussion of its variations compared to previous observations. Section 5 is devoted to the asteroseismological interpretation of our data. Section 6 summarizes our results.

\section{Observations and data reduction}

\subsection{Summary of previous work}

With a $T_{\text {eff }}=11440 \pm 350 \mathrm{~K}$ (Bergeron et al. 1995), HL Tau 76 is part of a group of ZZ Ceti stars located in the cool part of the instability strip, the red edge being defined by G30-20 and BPM24754 at $T_{\text {eff }}=11070 \pm 200 \mathrm{~K}$. Its $\log g=7.89 \pm$ 0.05 translates to a mass of $M / M_{\odot}=0.55 \pm 0.03$ assuming it has a pure carbon core, a helium layer fractional mass $M_{\mathrm{He}} / M_{\mathrm{WD}}=10^{-2}$ and a "thick" hydrogen layer fractional mass $M_{\mathrm{H}} / M_{\mathrm{WD}}=10^{-4}$, according to Bergeron et al. (1995) from the models of Wood (1995). However, since the hydrogen and helium mass fraction are not known, the derived total mass cannot be tightly constrained by spectroscopy alone.

HL Tau $76=$ V411 Tau is the very first discovered pulsating white dwarf (Landolt 1968). In the original discovery data set, Landolt identified a quasi-periodic variation of about $12.5 \mathrm{~min}$ with a large amplitude of 0.14 mag. This discovery was confirmed by observations of Warner \& Nather $(1970,1972)$ and the main period was more precisely determined as $12.437 \mathrm{~min}$. The light curve of Warner \& Nather (1972) was reanalysed by Page (1972) who established for the first time the 
multiperiodicity of HL Tau 76 in finding three periods: $748.5 \pm$ $2 \mathrm{~s}, 665 \pm 2 \mathrm{~s}$ and $628 \pm 2 \mathrm{~s}$. Later, Fitch (1973) obtained new observations confirming the multiperiodicity of HL Tau 76. However, while the largest amplitude was still at a period of $748.5 \mathrm{~s}$, another period of $494 \mathrm{~s}$ was present in the light curve, while the periods previously detected at $665 \mathrm{~s}$ and $628 \mathrm{~s}$ were absent, thus revealing the amplitude variations of HL Tau 76. Such amplitude variations were commonly found in many other ZZ Ceti stars discovered later on. HL Tau 76 has been further observed, either from single-site or from multi-site photometric campaigns, including a WET campaign (XCOV13) in February 1996, where it was a secondary target. A preliminary analysis of the data available until 1997 has been presented by Dolez \& Kleinman (1997). The available single-site data include those obtained at the $1.5 \mathrm{~m}$ telescope of the Tenerife Observatory in November 1989, at the $1.9 \mathrm{~m}$ telescope of the Haute Provence Observatory in December 1989 and at the 36-in telescope of the McDonald Observatory in October 1990. A short description of those observations is given in Dolez \& Kleinman (1997). In this paper, we will use those results when necessary. The frequency resolution achieved by these observations are $\approx 1.1 \mu \mathrm{Hz}$ in November $1989, \approx 9 \mu \mathrm{Hz}$ in December $1989, \approx 5.4 \mu \mathrm{Hz}$ in October 1990 and $2.6 \mu \mathrm{Hz}$ during XCOV13. In addition, a short run of time-resolved spectroscopy is described by Kotak et al. (2002b).

\subsection{The observations}

The observations described in this paper result from the Whole Earth Telescope multisite fast photometry campaign (XCOV18) organized in November 1999. During that campaign, HL Tau 76 was the first priority target.

The data have been obtained mostly with 3-channel photometers all equipped with blue sensitive photomultipliers (Hamamatsu R647-04 or similar) and used without a filter (white light). These instruments fulfill the specifications and requirements as prescribed by Kleinman et al. (1996). The sampling time was either $5 \mathrm{~s}$ or $10 \mathrm{~s}$. The sky background is continuously monitored by the third channel, with the target and comparison stars placed in the other two channels. Some data have been obtained with CCD photometers. Table 2 gives the Journal of the observations. The CCD data are identified in the last column.

During that campaign, the non-overlapping data resulted in a $66.5 \%$ duty cycle. The total duration of the campaign was 18 days. The data of 17 days were used for the computation of the power spectrum which leads to a frequency resolution in the Fourier Transform of the corresponding light curve $\approx 0.68 \mu \mathrm{Hz}$.

\subsection{Data reduction}

The photomultiplier (PMT) photometer data have been reduced in a now standard way (Nather et al. 1990; Kepler 1993). In 3-channel photometers, the sky background is measured at the beginning and at the end of each run in all channels. These data are used to determine the sensitivity ratios of the channels.
The sky background is subtracted point by point from the target and comparison star channels, after application of the proper sensitivity ratios. Each star channel is then corrected for extinction and normalized. When conditions show evidence for transparency variations, the normalized target star channel counts are divided by the smoothed comparison star channel counts. Subtracting unity from the resulting time series gives the time series on which the barycentric correction to the time base is applied. The exposure times of the PMT data were 5 or $10 \mathrm{~s}$ while in the case of the CCD data they were close to $30 \mathrm{~s}$. We performed some simulations to estimate the consequence of adding data sampled at different rates, which is the case for our data (PMT + CCD). We considered different combinations of parts of a noise free single sinusoidal light curve, with gaps, sampled at different rate $(5 \mathrm{~s}, 10 \mathrm{~s}, 20 \mathrm{~s})$ and calculated the corresponding window functions. This always results in a degraded window function showing higher amplitude aliases compared to the window function computed from the same light curve sampled at a uniform rate. As a result, we decided to homogenize the sampling rate by summing the PMT data to $30 \mathrm{~s}$, after having checked that there was no signals with periods shorter than $60 \mathrm{~s}$.

A few runs have been rejected where the noise level was too high, which was usually due to clouds or instrumental problems. In case of overlapping data, we tried to use the additional data to improve the signal/noise ratio. If a signal $S_{1}$, whose noise is $b_{1}$, overlaps with a signal $S_{2}$, whose noise is $b_{2}$, the weighted sum $\mathrm{S}$ of these signals is given by:

$S=S_{1} \times b_{2}^{2}\left(b_{1}^{2}+b_{2}^{2}\right)^{-1}+S_{2} \times b_{1}^{2}\left(b_{1}^{2}+b_{2}^{2}\right)^{-1}$.

The resulting noise $b$ is theoretically lower than the individual data noise by:

$b=b_{1} b_{2} / \sqrt{\left(b_{1}^{2}+b_{2}^{2}\right)}$.

This shows that by adding two signals of similar noise, the resulting noise may be improved by $\sqrt{2}$. Similarly, doing the weighted sum of signals whose noise differs by a factor of $3 \mathrm{im}-$ proves the resulting noise by only $5 \%$. This is only true in the ideal case where both signals are sampled at the same rate. Practically, we performed such a weighted sum only for data whose noise differs by no more than a factor of 3 . Otherwise, we kept only the best signal/noise ratio data. The resulting normalized light curve is shown in Fig. 1.

\section{The amplitude spectrum}

The amplitude spectrum of the time series is obtained by performing a Fourier Transform. As the amplitude spectrum of HL Tau 76 is somewhat complex, it is shown on a large scale in three parts in Figs. 2-4 for the frequency intervals $0-2000 \mu \mathrm{Hz}, 2000-4000 \mu \mathrm{Hz}$ and $4000-6000 \mu \mathrm{Hz}$ respectively. The amplitude spectrum above $6000 \mu \mathrm{Hz}$ is not shown as it is mainly noise and shows only very low amplitude insignificant signal. Each figure is formed of 5 panels, each covering a $400 \mu \mathrm{Hz}$ frequency interval. The corresponding window function is shown in Fig. 5 in full size and on a larger scale for a better visibility of the remaining aliases. 


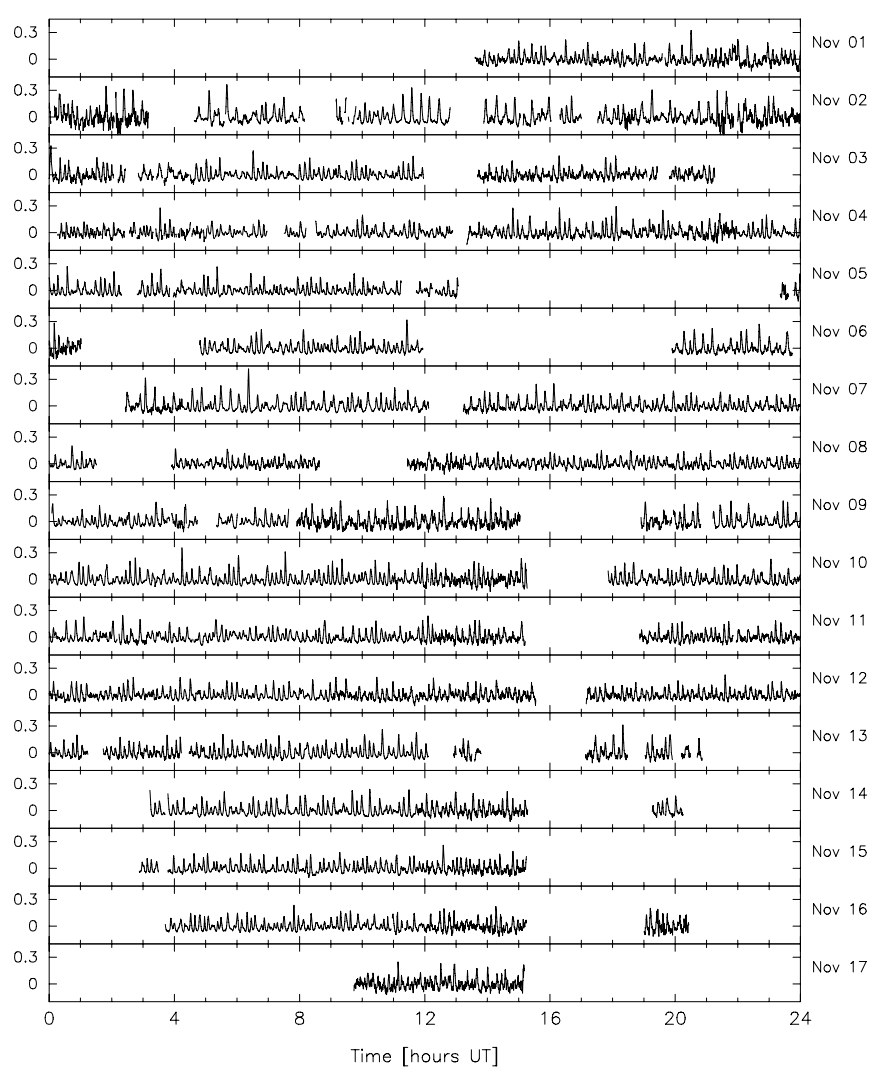

Fig. 1. Normalized light curve of HL Tau 76 during the 1999 WET campaign (XCOV18). All the runs used in the power spectrum computation are shown. We subtract unity to the normalized light curve so that its average value is 0 . The fractional modulation intensity (mi) is plotted as a function of time (UT)(left side scale). Each panel corresponds to one day (right side scale).

The peaks in the amplitude spectrum are extracted by successive non-linear least squares fits followed by prewhitening The significance of the peaks is determined in the following way. For each peak, we estimate the average noise $\sigma$ on a frequency interval of $100 \mu \mathrm{Hz}$ centered on the peak frequency. The False Alarm Probability (FAP) (Scargle 1982) for that peak to be due to noise on such a frequency interval is estimated. As a first step, all peaks with a FAP $\leq 10^{-3}$ are considered as significant, which is equivalent to considering as significant all peaks with amplitude larger than $3.4 \times \sigma$. Each of these frequencies was then subtracted from the light curve in successive steps of pre-whitening, the whole FT being recomputed at each step.

An illustration of the frequency iterative extraction process is shown in Fig. 6 for the first 6 frequencies. We can see in the panel hl07 that after the extraction of the 6 largest amplitude peaks, a large number of significant peaks are still present. The last panel shows the residuals after pre-whitening by the first 52 frequencies. The remaining peaks are all linear combinations of the previously extracted frequencies. This procedure extracts a total number of 85 significant peaks which are listed in Table 3. However, as a second step, one increases the detection limit to $4.1 \times \sigma$. This is equivalent to estimate as significant all peaks with a $\mathrm{FAP} \leq 10^{-3}$ on the total frequency range of the FT up to the Nyquist frequency. This more severe
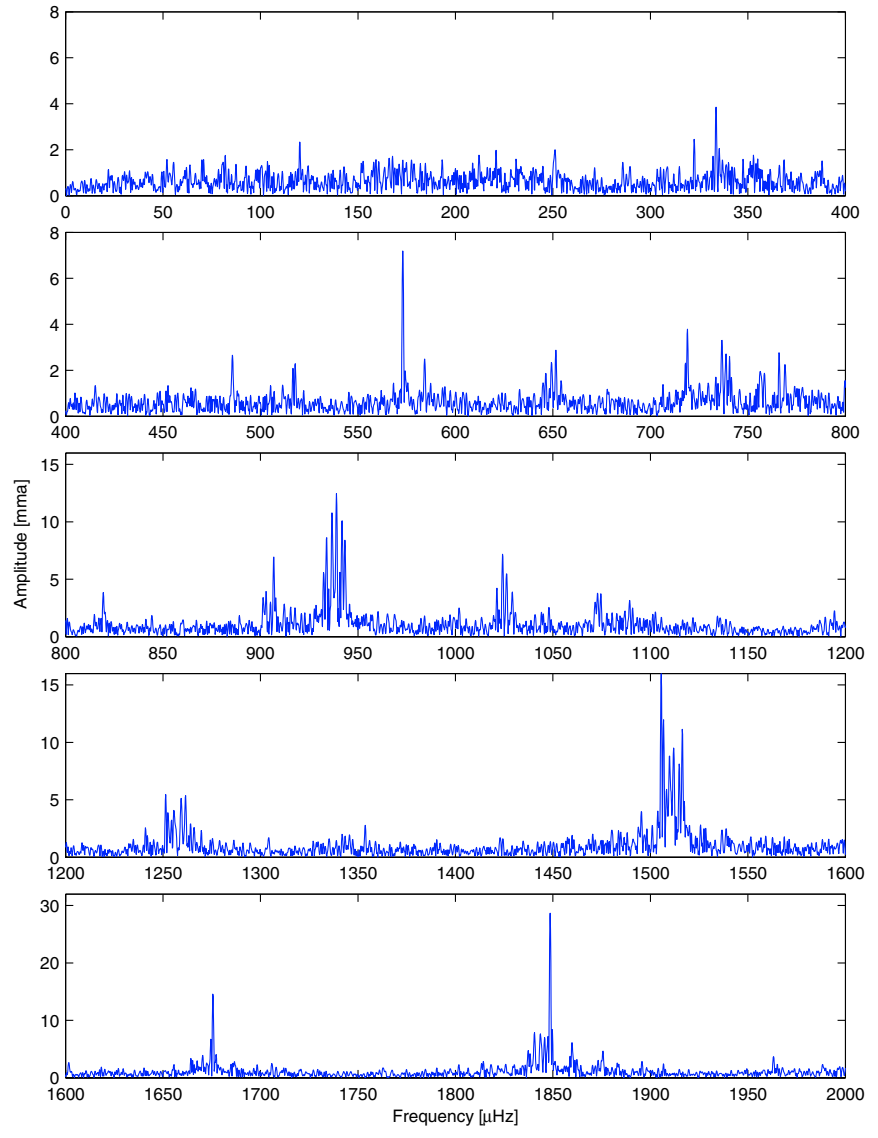

Fig. 2. Fourier amplitude spectrum of HL Tau 76 in the range 0 to $2000 \mu \mathrm{Hz}$. The amplitude expressed in milli-modulation amplitude (mma) is plotted as a function of the frequency expressed in $\mu \mathrm{Hz}$. Note that the vertical scale for the amplitude varies for each panel.

selection criterion eliminates 7 of the peaks previously selected whose significance is consequently dubious. Those peaks are noted by a colon (:) in Table 3. Five of them will be identified as linear combinations below. Their elimination does not affect the following discussion. This is not the case for the remaining two (peaks 13 and 26 in Table 3) which were considered as independent frequencies after the first step. To be conservative, we will not use them in the proposed asteroseismological interpretation. We are then left with 78 significant peaks.

Table 3 gives the list of the frequencies in Col. 2. The third column gives $\delta f$, the formal error on the frequencies derived from the least-squares analysis. The fourth and fifth columns give the corresponding periods and their uncertainties. The sixth column gives the amplitude in units of millimodulation amplitude (mma). The phases derived from the least-squares fits are given in the seventh column (between 0 and 1 for the interval 0 to $2 \pi$ ). The formal errors estimated in this way are lower limits to the true uncertainties. Another way of estimating the uncertainty on the frequency determination consists in using the frequencies of the linear combination peaks. As discussed below, a number of peaks in the Fourier spectrum are linear combinations of higher amplitude peaks. The differences $\Delta f$ between the observed frequency of these combination peaks and the frequency resulting from the sum or the difference of their "parent" peaks frequency is a 

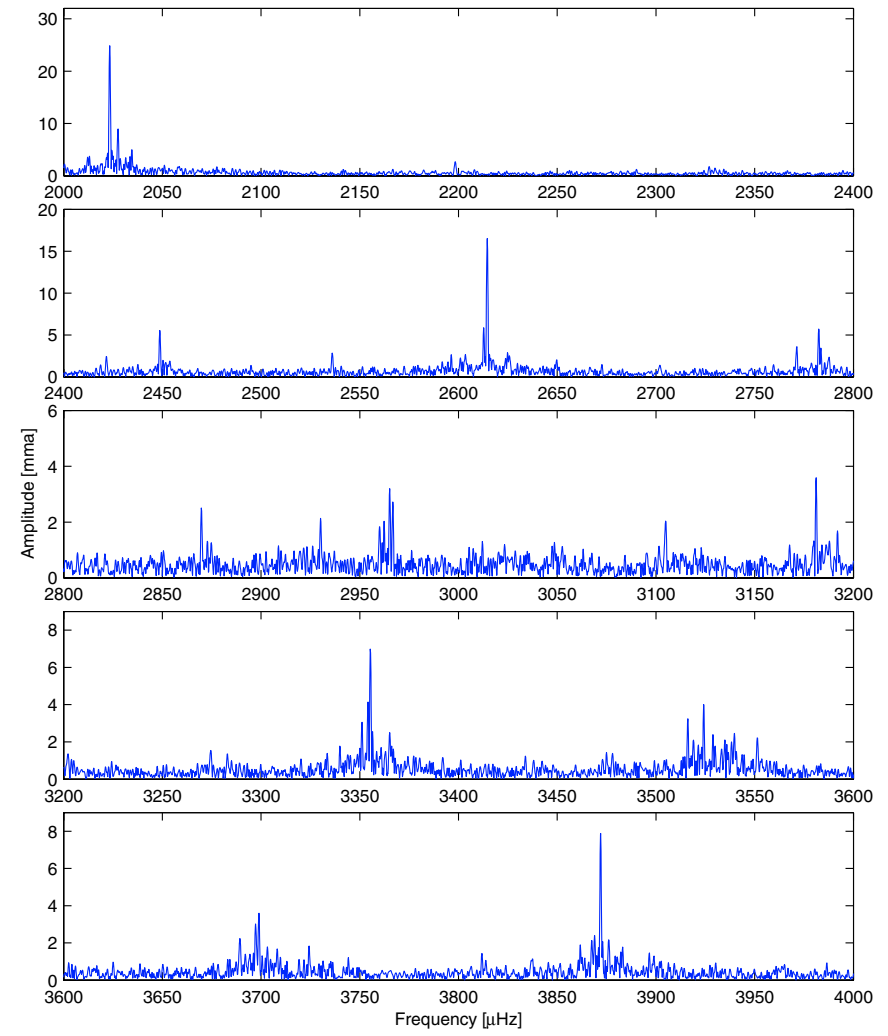

Fig. 3. Same as Fig. 2 for the frequency range 2000 to $4000 \mu \mathrm{Hz}$.

measure of the "true" uncertainty on the frequency determination. The linear combinations are identified in the eighth column and $\Delta f$ is given in the last column of Table 3 . The average $\delta f$ is $0.028 \mu \mathrm{Hz}$ and the average $\Delta f$ is $0.038 \mu \mathrm{Hz}$.

The amplitude spectrum is characterized by a small number of large amplitude peaks, with amplitudes exceeding $10 \mathrm{mma}$, plus a large number of smaller amplitude peaks. It is immediately clear that many of these small amplitude peaks are linear combinations and/or harmonics of the large amplitude ones. These frequency combinations reflect the nonlinear response of the flux perturbations at the level of the photosphere to the sinusoidal perturbations at the bottom of the convection zone. This is a consequence of the variations of the depth of the convection zone and of the correlated convective flux during the pulsations. These nonlinearities induce the non-sinusoidal nature of the pulse shapes, which in turn materialize in the Fourier spectrum by the occurence of combination frequencies and harmonics. This is a frequent property of the cool DAV Fourier amplitude spectra. As always in such cases, one finds frequency combinations such that:

$f_{1} \pm f_{2}=f_{3}$,

with no a priori knowledge of which are the "parent" frequencies and which is the "combination".

To select which are the most probable linear combinations, we used the following arguments: i) the comparison with other epochs of data to check that modes may show up without their linear combinations while the contrary is excluded; ii) the combination of two multiplets results in a much different fine structure than each parent multiplet; iii) the amplitude of the

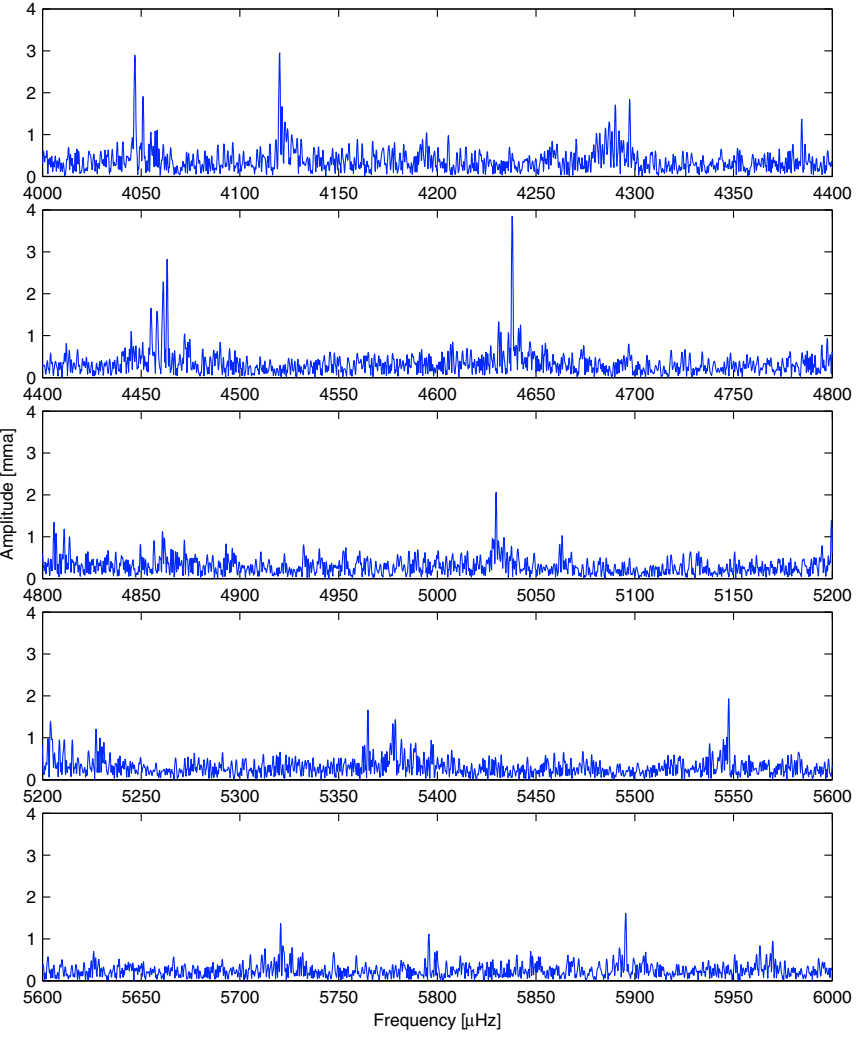

Fig. 4. Same as Fig. 2 for the range 4000 to $6000 \mu \mathrm{Hz}$.
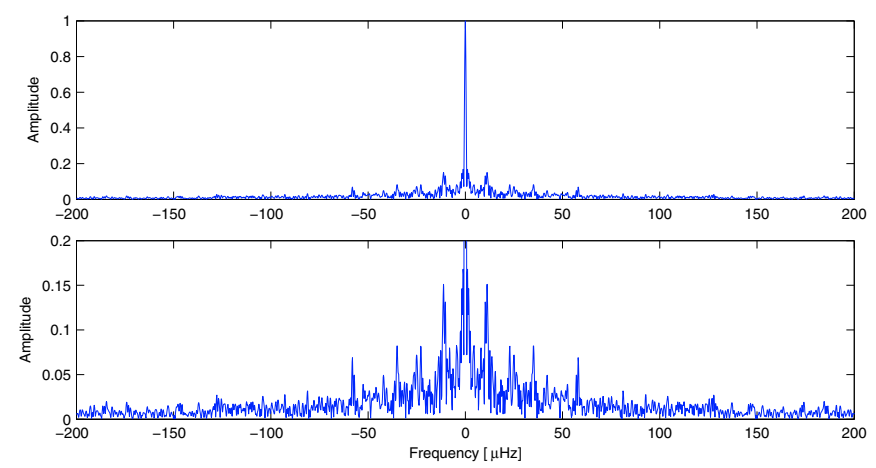

Fig. 5. Window function obtained during the 1999 WET campaign. The window function is shown on the same frequency scale than the Fourier amplitude spectrum. The upper panel shows the window function normalized to an amplitude of unity at maximum. The lower panel shows the same window function truncated at an amplitude of 0.2 for a better visibility of the remaining aliases

combinations is generally smaller than the amplitude of their parent modes; iv) combinations of combinations are less likely than "first order" combinations; and v) if one knows the period spacings one can check whether a mode is at the expected frequency, which is an indication that it may rather be a real mode. We made use of these criteria to select the linear combinations in the list of frequencies given in Table 3.

We started our search for linear combinations by looking for peaks whose frequency corresponds to the sums or the differences of frequencies of other peaks within $\approx$ two formal errors of the frequency determination as obtained from the 


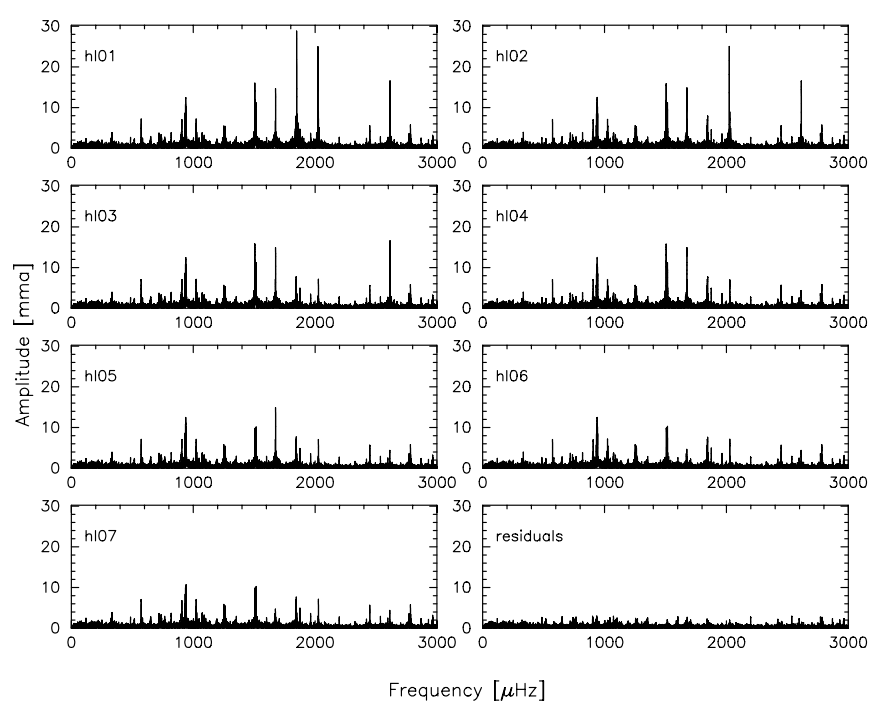

Fig. 6. Illustration of the frequency extraction process. The successive steps of prewhitening are shown for the first six frequencies. Each panel shows the Fourier amplitude spectrum, in unit of mma, in the frequency interval $0-3000 \mu \mathrm{Hz}$. The panels are labelled from hl01 to hl07. The panel hl01 is the full Fourier spectrum. The next panel, hl02, is the Fourier spectrum after prewhitening by the largest amplitude peak at $1848 \mu \mathrm{Hz}$. The next panel, hl03 is the Fourier spectrum after prewhitening by the two largest amplitude peaks at $1848 \mu \mathrm{Hz}$ and $2023 \mu \mathrm{Hz}$, etc. The last panel shows the residuals after prewhitening by the first 52 largest amplitude frequencies. All the remaining low amplitude but still significant peaks are linear combination of the previously extracted largest amplitude peaks.

least-squares fit. By applying these rules, we find more than one set of two "parent" frequencies for almost every linear combination. To select the most probable "parent" combination, we used one or more of the following additional criteria: i) the sum (difference) of the "parent" frequencies is the closest to the frequency of the resulting combination; or ii) the selected "parent" peaks have a larger amplitude than the other "parent" candidates; or iii) a quadratic linear combination of two frequencies is more probable than a higher degree combination involving more than two parent frequencies (cubic and higher). The first harmonics of the four largest amplitude peaks are also found but, with one exception, they overlap with another linear combination so that the resulting frequency does not match exactly the expected harmonics frequency. That is case for the peak 62 which is at $1.75 \mu \mathrm{Hz}$ of the first harmonics of the peak 44 and is also the combination of peak 39 and 48 . The exception is the peak 67 which is exactly the first harmonics of the peak 48 . The peak 79 is at $1.95 \mu \mathrm{Hz}$ of the first harmonics of the peak 53 but is also the (less probable) cubic linear combination of the peaks 11, 39 and 53. Finally, the peak 59 is at $2.7 \mu \mathrm{Hz}$ of the first harmonics of the peak 39 , but is also the quadratic linear combination of the peaks 29 and 44 . It must be noted that some linear combinations resulting from large amplitude peaks do have themselves an amplitude large enough to produce higher order linear combinations with their former "parent" peaks or with other large amplitude peaks. It is one of the reasons why the power spectrum has such a complex structure. One example of such a hierarchical series of linear combinations is the combination of the two largest amplitude modes at $1848.58 \mu \mathrm{Hz}$ (44) and at $2023.36 \mu \mathrm{Hz}$ (48) which produces a quadratic linear combination at $3871.96 \mu \mathrm{Hz}(65)$ of $8.0 \mathrm{mma}$ amplitude. This resulting frequency combines with the fourth largest amplitude peak (39) to produce the cubic linear combination at $5547.46 \mu \mathrm{Hz}$ (peak 79). Another interesting case is the peak 83 resulting from a combination of the first harmonics of the largest amplitude peak (44) with the second largest amplitude peak (48). Table 3 lists the selected most probable linear combination in Col. 7. As a summary, among the listed 85 significant peaks (below $6000 \mu \mathrm{Hz}$ ) we find 31 quadratic combinations, of which 4 are also first harmonics of the largest amplitude peaks, and 19 cubic combinations.

Since the non-linear least squares fit used to derive the frequencies and amplitudes from the light curve also gives the phases, one could anticipate using the analytical relation between the phases of the parent modes and the phase of their linear combination (Wu 2001) to check whether the combinations have been correctly identified. The theory developed by $\mathrm{Wu}$ (2001), after Brickhill, relies on the assumption that the convective turn-over time-scale is much shorter than the pulsation periods. It describes how the flux perturbations at the photosphere is related to the flux perturbations at the bottom of the convection zone. In the case of a linear combination of two modes, the phase delay between the two parent modes and their linear combination implies a thermal time constant $\tau_{c 0}$ which is related to the thermal relaxation time scale of the convection zone and to the characteristic time scales of the response of the photosphere and of the superadiabatic region towards gravity-mode pulsations. With the adopted ML2/ $\alpha=$ 0.6 version of the mixing-length theory, after Bergeron et al. (1995), the thermal relaxation time scale of the convection zone in HL Tau 76 is of the same order, $\approx 300 \mathrm{~s}$, than the pulsation periods. The response of the photosphere to the flux perturbations at the bottom of the convection zone is not straightforward in this case. The potentially useful informations given by the phases of the linear combinations compared to the phases of their parent modes is not easy to interpret in the case of HL Tau 76. It is why, while those phases are given in Table 3, they are not used in the present paper to check the validity of our linear combination identification.

\section{Analysis of the amplitude spectrum}

Having eliminated the doubtful peaks and the linear combinations, one is left with 33 frequencies which have to be considered as independent frequencies present in the star. This makes HL Tau 76 the next richest pulsator after PG 1159-035 (Winget et al. 1991), GD 358 (Winget et al. 1994; Kepler et al. 2003) and RX J2117+3412 (Vauclair et al. 2002). These frequencies appear in 10 separated groups between $718.99 \mu \mathrm{Hz}$ and $2614.55 \mu \mathrm{Hz}$. All the higher frequency peaks in XCOV18 are identified as linear combinations (quadratic and higher) of lower frequency peaks. In their analysis of the XCOV13 data Dolez \& Kleinman (1997) also conclude that all the peaks with frequency above $2614 \mu \mathrm{Hz}$ are linear combination peaks. 
Table 3. List of the frequencies identified in HL Tau 76.

\begin{tabular}{|c|c|c|c|c|c|c|c|c|}
\hline$n$ & $\begin{array}{c}\text { Frequency } \\
{[\mu \mathrm{Hz}]}\end{array}$ & $\begin{array}{c}\delta f \\
{[\mu \mathrm{Hz}]}\end{array}$ & $\begin{array}{c}\text { Period } \\
{[\mathrm{s}]}\end{array}$ & $\begin{array}{l}\delta P \\
{[\mathrm{~s}]}\end{array}$ & $\begin{array}{c}A \pm 0.26 \\
{[\mathrm{mma}]}\end{array}$ & Phase & $\begin{array}{c}\text { linear } \\
\text { combination }\end{array}$ & $\begin{array}{c}\Delta f \\
{[\mu \mathrm{Hz}]}\end{array}$ \\
\hline 1 & 333.68 & 0.026 & 2996.88 & 0.234 & 3.83 & 0.7481 & $44-35$ & 0.01 \\
\hline 2 & 572.99 & 0.015 & 1745.23 & 0.045 & 7.02 & 0.7008 & $30-10$ & 0.03 \\
\hline 3 & 718.99 & 0.028 & 1390.84 & 0.054 & 3.62 & 0.1139 & & \\
\hline 4 & 736.67: & 0.033 & 1357.46 & 0.061 & 3.14 & 0.3150 & $37-10$ & 0.09 \\
\hline 5 & 765.96 & 0.036 & 1305.55 & 0.060 & 2.68 & 0.3277 & $53-44$ & 0.01 \\
\hline 6 & 819.34 & 0.029 & 1220.49 & 0.043 & 3.48 & 0.4134 & $41-17$ & 0.06 \\
\hline 7 & 901.44 & 0.030 & 1109.33 & 0.036 & 3.31 & 0.0941 & $41-14$ & 0.23 \\
\hline 8 & 906.72 & 0.017 & 1102.87 & 0.020 & 6.22 & 0.2139 & $44-14$ & 0.05 \\
\hline 9 & 932.40 & 0.019 & 1072.50 & 0.021 & 5.19 & 0.2807 & $15+29-36$ & 0.08 \\
\hline 10 & 933.87 & 0.010 & 1070.81 & 0.011 & 9.61 & 0.5495 & & \\
\hline 11 & 936.73 & 0.010 & 1067.54 & 0.011 & 9.74 & 0.1600 & & \\
\hline 12 & 938.99 & 0.009 & 1064.97 & 0.011 & 11.30 & 0.7761 & $53-39$ & 0.05 \\
\hline 13 & 941.09: & 0.030 & 1062.60 & 0.033 & 3.23 & 0.2608 & & \\
\hline 14 & 941.80 & 0.009 & 1061.80 & 0.010 & 11.44 & 0.3381 & & \\
\hline 15 & 943.20 & 0.012 & 1060.22 & 0.013 & 7.67 & 0.5337 & & \\
\hline 16 & 1021.26 & 0.024 & 979.18 & 0.022 & 4.34 & 0.3558 & & \\
\hline 17 & 1024.19 & 0.016 & 976.38 & 0.015 & 6.46 & 0.0317 & & \\
\hline 18 & 1026.23 & 0.020 & 974.44 & 0.019 & 4.96 & 0.4627 & & \\
\hline 19 & 1029.23 & 0.026 & 971.60 & 0.024 & 3.89 & 0.1976 & & \\
\hline 20 & 1071.58 & 0.032 & 933.20 & 0.027 & 3.22 & 0.3445 & & \\
\hline 21 & 1074.52 & 0.028 & 930.64 & 0.024 & 3.62 & 0.3974 & & \\
\hline 22 & 1089.33: & 0.034 & 917.99 & 0.028 & 3.09 & 0.6275 & $48-10$ & 0.156 \\
\hline 23 & 1251.40 & 0.020 & 799.10 & 0.012 & 5.19 & 0.4890 & & \\
\hline 24 & 1252.64 & 0.025 & 798.31 & 0.016 & 4.04 & 0.1624 & & \\
\hline 25 & 1255.69 & 0.027 & 796.37 & 0.017 & 3.74 & 0.3478 & & \\
\hline 26 & 1256.69: & 0.029 & 795.74 & 0.018 & 3.55 & 0.2307 & & \\
\hline 27 & 1259.31 & 0.020 & 794.08 & 0.012 & 5.10 & 0.5619 & & \\
\hline 28 & 1261.50 & 0.025 & 792.70 & 0.016 & 4.08 & 0.1001 & & \\
\hline 29 & 1505.54 & 0.007 & 664.21 & 0.003 & 14.99 & 0.3064 & & \\
\hline 30 & 1506.83 & 0.008 & 663.64 & 0.003 & 11.90 & 0.8742 & & \\
\hline 31 & 1508.71 & 0.027 & 662.81 & 0.011 & 3.49 & 0.6391 & & \\
\hline 32 & 1509.83 & 0.009 & 662.32 & 0.004 & 10.84 & 0.5580 & & \\
\hline 33 & 1510.77 & 0.013 & 661.91 & 0.005 & 7.61 & 0.1502 & & \\
\hline 34 & 1511.96 & 0.011 & 661.39 & 0.005 & 9.30 & 0.0849 & & \\
\hline 35 & 1514.91 & 0.013 & 660.10 & 0.005 & 7.35 & 0.7732 & & \\
\hline 36 & 1516.26 & 0.009 & 659.51 & 0.004 & 10.71 & 0.9114 & & \\
\hline 37 & 1670.45 & 0.029 & 598.64 & 0.010 & 3.55 & 0.4324 & & \\
\hline 38 & 1674.63 & 0.022 & 597.14 & 0.008 & 4.48 & 0.9758 & & \\
\hline 39 & 1675.61 & 0.007 & 596.79 & 0.002 & 14.60 & 0.2749 & & \\
\hline 40 & 1840.56 & 0.018 & 543.31 & 0.005 & 5.57 & 0.8478 & $10-14+44$ & 0.04 \\
\hline 41 & 1843.48 & 0.015 & 542.45 & 0.004 & 6.95 & 0.9503 & & \\
\hline 42 & 1844.61 & 0.025 & 542.12 & 0.007 & 3.99 & 0.7636 & $30+44-33$ & 0.03 \\
\hline 43 & 1845.74 & 0.013 & 541.78 & 0.004 & 8.05 & 0.5639 & & \\
\hline
\end{tabular}


Table 3. continued.

\begin{tabular}{|c|c|c|c|c|c|c|c|c|}
\hline$n$ & $\begin{array}{c}\text { Frequency } \\
{[\mu \mathrm{Hz}]}\end{array}$ & $\begin{array}{c}\delta f \\
{[\mu \mathrm{Hz}]}\end{array}$ & $\begin{array}{c}\text { Period } \\
{[\mathrm{s}]}\end{array}$ & $\begin{array}{l}\delta P \\
{[\mathrm{~s}]}\end{array}$ & $\begin{array}{c}A \pm 0.26 \\
{[\mathrm{mma}]}\end{array}$ & Phase & $\begin{array}{c}\text { linear } \\
\text { combination }\end{array}$ & $\begin{array}{c}\Delta f \\
{[\mu \mathrm{Hz}]}\end{array}$ \\
\hline 44 & 1848.58 & 0.003 & 540.95 & 0.001 & 28.45 & 0.1751 & & \\
\hline 45 & 1849.87 & 0.022 & 540.57 & 0.006 & 4.40 & 0.7465 & $30+44-29$ & 0.00 \\
\hline 46 & 1875.77 & 0.025 & 533.11 & 0.007 & 3.98 & 0.4290 & $10+14$ & 0.10 \\
\hline 47 & 1963.15 & 0.030 & 509.38 & 0.008 & 3.40 & 0.2265 & $14+16$ & 0.08 \\
\hline 48 & 2023.36 & 0.004 & 494.22 & 0.001 & 4.42 & 0.8567 & & \\
\hline 49 & 2027.53 & 0.014 & 493.21 & 0.003 & 7.12 & 0.7159 & & \\
\hline 50 & 2198.32: & 0.035 & 454.89 & 0.007 & 2.78 & 0.3055 & $11+28$ & 0.07 \\
\hline 51 & 2448.74 & 0.018 & 408.37 & 0.003 & 5.62 & 0.7558 & $15+29$ & 0.00 \\
\hline 52 & 2612.58 & 0.022 & 382.76 & 0.003 & 4.50 & 0.7803 & $11+39$ & 0.23 \\
\hline 53 & 2614.55 & 0.006 & 382.47 & 0.001 & 16.47 & 0.5812 & & \\
\hline 54 & 2782.47 & 0.018 & 359.39 & 0.002 & 5.69 & 0.7640 & $10+44$ & 0.02 \\
\hline 55 & 2965.17: & 0.030 & 337.24 & 0.003 & 3.18 & 0.6527 & $14+48$ & 0.01 \\
\hline 56 & 3104.91 & 0.044 & 322.07 & 0.003 & 2.04 & 0.0194 & $28+41$ & 0.08 \\
\hline 57 & 3181.10 & 0.033 & 314.35 & 0.004 & 3.68 & 0.9605 & $29+39$ & 0.05 \\
\hline 58 & 3274.64 & 0.049 & 305.37 & 0.005 & 1.60 & 0.1444 & $23+48$ & 0.12 \\
\hline 59 & 3353.96: & 0.033 & 298.15 & 0.003 & 3.60 & 0.8302 & $29+44$ & 0.15 \\
\hline 60 & 3355.37 & 0.015 & 298.03 & 0.001 & 7.08 & 0.4778 & $30+44$ & 0.04 \\
\hline 61 & 3524.18 & 0.023 & 283.75 & 0.001 & 4.06 & 0.8590 & $39+44$ & 0.01 \\
\hline 62 & 3698.91 & 0.033 & 270.35 & 0.002 & 3.73 & 0.5215 & $39+48$ & 0.06 \\
\hline 63 & 3724.34 & 0.047 & 268.50 & 0.003 & 1.74 & 0.0055 & $11+15+41$ & 0.92 \\
\hline 64 & 3811.80 & 0.054 & 262.34 & 0.004 & 1.35 & 0.4416 & $13+16+44$ & 0.86 \\
\hline 65 & 3871.96 & 0.014 & 258.26 & 0.001 & 8.00 & 0.5168 & $44+48$ & 0.02 \\
\hline 66 & 3876.06 & 0.049 & 257.99 & 0.003 & 1.62 & 0.2944 & $44+49$ & 0.05 \\
\hline 67 & 4046.80 & 0.034 & 247.10 & 0.002 & 2.84 & 0.2555 & $2 \times 48$ & 0.08 \\
\hline 68 & 4050.98 & 0.046 & 246.85 & 0.003 & 1.84 & 0.1111 & $48+49$ & 0.09 \\
\hline 69 & 4120.14 & 0.034 & 242.71 & 0.002 & 2.88 & 0.4000 & $29+53$ & 0.05 \\
\hline 70 & 4292.04 & 0.058 & 232.98 & 0.003 & 1.06 & 0.1166 & $14+38+39$ & 0.01 \\
\hline 71 & 4297.37 & 0.040 & 232.70 & 0.003 & 1.82 & 0.3944 & $10+35+44$ & 0.01 \\
\hline 72 & 4455.00 & 0.048 & 224.46 & 0.003 & 1.66 & 0.2486 & $13+37+41$ & 0.03 \\
\hline 73 & 4458.02 & 0.050 & 224.31 & 0.002 & 1.52 & 0.4555 & $41+53$ & 0.01 \\
\hline 74 & 4461.19 & 0.038 & 224.15 & 0.002 & 2.42 & 0.4500 & $43+53$ & 0.90 \\
\hline 75 & 4637.91 & 0.025 & 215.61 & 0.001 & 3.96 & 0.8972 & $48+53$ & 0.00 \\
\hline 76 & 5029.76 & 0.044 & 198.81 & 0.002 & 2.06 & 0.1555 & $29+39+44$ & 0.02 \\
\hline 77 & 5199.63 & 0.052 & 192.32 & 0.002 & 1.37 & 0.3944 & $30+41+44$ & 0.73 \\
\hline 78 & 5204.10 & 0.052 & 192.15 & 0.002 & 1.36 & 0.1236 & $32+43+44$ & 0.05 \\
\hline 79 & 5227.15 & 0.056 & 191.30 & 0.002 & 1.24 & 0.3805 & $11+39+53$ & 0.24 \\
\hline 80 & 5364.76 & 0.048 & 186.40 & 0.001 & 1.70 & 0.1972 & $39+41+43$ & 0.08 \\
\hline 81 & 5378.73 & 0.052 & 185.91 & 0.001 & 1.40 & 0.2750 & $30+44+48$ & 0.05 \\
\hline 82 & 5547.46 & 0.045 & 180.26 & 0.001 & 1.90 & 0.1069 & $39+44+48$ & 0.09 \\
\hline 83 & 5720.65 & 0.055 & 174.80 & 0.001 & 1.34 & 0.2166 & $2 \times 44+48$ & 0.13 \\
\hline 84 & 5795.72 & 0.060 & 172.54 & 0.002 & 1.06 & 0.4875 & $29+39+53$ & 0.01 \\
\hline 85 & 5895.42 & 0.049 & 169.62 & 0.001 & 1.60 & 0.4000 & $26+48+53$ & 0.81 \\
\hline
\end{tabular}

\subsection{Time variations}

The observations of the HL Tau 76 pulsations span a total interval of 35 years between their discovery and the present
WET campaign. They provide some information on the star's amplitude and/or frequency variations. Such information is of interest to understand the physics underlying the excitation mechanism. It is also important to check whether there are 
pulsations modes stable enough in amplitude and frequency to be suitable for $\dot{P}$ measurements. Such $\dot{P}$ measurements can be used to estimate the cooling time and constrain the core composition (see Kepler et al. 2000a).

The available observations consist in part of: the original discovery of the HL Tau 76 variability by Landolt (1968), the observations themselves having been carried out in 1964; the confirmation data and additional data by Warner \& Nather (1970, 1972), the later ones reanalysed by Page (1972); the data published by Fitch (1973). It seems that the star was forgotten for some time until new observations, making use of 3-channel photometers, were undertaken through single-site or multi-site campaigns. These new observations, including a WET campaign (XCOV13) during which HL Tau was a second priority target, have been analysed by Dolez \& Kleinman (1997).

Landolt's discovery light curve was dominated by a $746 \mathrm{~s}$ period (1340 $\mu \mathrm{Hz}$ frequency) of $0.14 \mathrm{mag}$ amplitude. Later on, Page (1972) identified three frequencies in the Warner \& Nather (1972) light curve, at $1341 \mu \mathrm{Hz}$ (746 s), $1504 \mu \mathrm{Hz}$ (665 s) and $1592 \mu \mathrm{Hz}$ (628 s). In his data, Fitch (1973) found two frequencies at $1340 \mu \mathrm{Hz}$ (746 s) and $2023 \mu \mathrm{Hz}$ (494 s). During these early days, HL Tau 76 was pulsating with a preferred frequency around $1340 \mu \mathrm{Hz}$. This frequency, as well as the $1592 \mu \mathrm{Hz}$, have not been observed since, or at least had amplitudes below detection limits. From the frequencies observed in those early data, only those at $1504 \mu \mathrm{Hz}$ and $2023 \mu \mathrm{Hz}$ are still detected in the data analysed by Dolez \& Kleinman (1997) and in the present WET data.

Because the amplitude spectrum of HL Tau 76 is so rich, one must be careful when comparing spectra of different frequency resolutions to infer amplitude and/or frequency variations. As can be seen from Table 3, some frequency regions contain a number of closely-spaced frequency peaks which could not be resolved in short data sets. For instance, the timeresolved spectroscopy observations presented by Kotak et al. (2002b) are too short to resolve most of the complex structures seen in the amplitude spectrum. While this method is supposed to be insensitive to the azimuthal degree, yet it is necessary to resolve the modes of different $\ell$ degrees which may overlap in frequency. This case occurs in HL Tau 76 as discussed in the next section. The only comparison one can attempt here is with the data of the 1996 WET campaign (XCOV13). The frequency resolution of the amplitude spectrum achieved by XCOV13 is about $2.6 \mu \mathrm{Hz}$. The formal error on the frequencies of the largest amplitude peaks, derived from the least-squares fit, is of the order of $0.01 \mu \mathrm{Hz}$. By comparison, the frequency resolution achieved by the present XCOV18 data set is $0.65 \mu \mathrm{Hz}$, 4 times better. Considering the frequency differences of the peaks listed in Table 3, one concludes that most of them, if present during XCOV13, should have been resolved. So, it is meaningful to compare both WET campaigns. This comparison gives us some information on the variability of the power spectrum's fine structure on a 3.7 year time scale. The amplitudes and frequencies of the modes in common between XCOV13 and XCOV18 are given in Table 4. Some cases require specific comments.

The $741.9 \mu \mathrm{Hz}$ peak seen in XCOV13 and not detected in XCOV18 and the $736.7 \mu \mathrm{Hz}$ marginally detected in
Table 4. Amplitude variations in HL Tau 76 between XCOV13 and XCOV 18 .

\begin{tabular}{cccc}
\hline \hline $\begin{array}{c}\text { Frequency } \\
{[\mu \mathrm{Hz}]} \\
\text { XCOV13 }\end{array}$ & $\begin{array}{c}\text { Amplitude } \\
{[\mathrm{mma}]} \\
\text { XCOV13 }\end{array}$ & $\begin{array}{c}\text { Frequency } \\
{[\mu \mathrm{Hz}]} \\
\text { XCOV18 }\end{array}$ & $\begin{array}{c}\text { Amplitude } \\
{[\mathrm{mma}]} \\
\text { XCOV18 }\end{array}$ \\
\hline 741.9 & 9.2 & 736.7 & 3.1 \\
939.4 & 18.3 & 939.0 & 11.3 \\
1072.4 & 23.2 & 1071.6 & 3.2 \\
1255.5 & 8.9 & 1255.7 & 3.7 \\
1280.3 & 9.1 & abs & abs \\
1521.0 & 10.3 & 1516.3 & 10.7 \\
1675.0 & 14.4 & 1675.6 & 14.6 \\
1848.3 & 37.3 & 1848.6 & 28.4 \\
2023.4 & 27.8 & 2023.4 & 24.4 \\
2223.3 & 6.7 & abs & abs \\
2614.6 & 20.1 & 2614.5 & 16.5 \\
\hline
\end{tabular}

XCOV18 are probably two components of a same multiplet (see discussion in 5.2.4 below).

Dolez \& Kleinman (1997) considered the peak at $939.4 \mu \mathrm{Hz}$ as a linear combination between their $2614 \mu \mathrm{Hz}$ and $1675 \mu \mathrm{Hz}$ peaks. We also suggest that this peak may be a linear combination of the same peaks but on a different basis; namely, in XCOV18, both the $1675.61 \mu \mathrm{Hz}$ and the $2614.55 \mu \mathrm{Hz}$ peaks are present and have amplitudes larger than the $938.99 \mu \mathrm{Hz}$ peak (14.6 mma, $16.47 \mathrm{mma}$ and $11.30 \mathrm{mma}$ respectively). On this argument we suggest that $938.99 \mu \mathrm{Hz}$ peak may be a linear combination of the two other peaks. In contrast, during XCOV13, the $939.4 \mu \mathrm{Hz}$ peak amplitude was intermediate between the amplitudes of the two other peaks. Applying the selection rule used in the present paper to the XCOV13 data, we would have concluded that it is the $1675 \mu \mathrm{Hz}$ peak which is a linear combination of the two others. This illustrates the ambiguous identification of the "true" stellar independent pulsation frequencies versus their linear combinations. In this particular case, one sees that the amplitude of the $1675 \mu \mathrm{Hz}$ peak did not change from XCOV13 to XCOV18 (14.4 mma and $14.60 \mathrm{mma}$ respectively) while the $2614 \mu \mathrm{Hz}$ peak decreased its amplitude from $20.1 \mathrm{mma}$ during XCOV13 to $16.47 \mathrm{mma}$ during XCOV18. The $939 \mu \mathrm{Hz}$ peak decreased in amplitude also from $18.3 \mathrm{mma}$ during XCOV13 to 11.30 during XCOV18. If the $1675 \mu \mathrm{Hz}$ was the resultant linear combination, one would not expect its amplitude to remain constant while its two presumable "parent" peaks decreased in amplitude. To solve the puzzling nature of this $939 \mu \mathrm{Hz}$ frequency, we looked back into the unpublished earlier single-site data taken in November and December 1989 and in October 1990. We find that the three frequencies are always detected except the $939 \mu \mathrm{Hz}$ peak in December 1989 . This suggests that the two other frequencies must be independent modes. In the other runs, both the $939 \mu \mathrm{Hz}$ and the $2614 \mu \mathrm{Hz}$ peaks had the highest amplitude in turn. These data suggest that the three frequencies may correspond to three independent modes. In that case, they would not be simply 
linked through a linear combination but could form a true resonance. We will check below whether there are modes expected by the asymptotic theory at those frequencies.

The peak at $1072 \mu \mathrm{Hz}$ experienced a spectacular decrease of its amplitude. In XCOV18, this peak is resolved into a doublet, but both components have much lower amplitude: 3.22 and 3.62 mma respectively, than the peak seen during XCOV13. One of the peaks, at least, must have changed significantly in amplitude between the two runs. The $1255.5 \mu \mathrm{Hz}$ peak seen in XCOV13 with a 8.9 mma amplitude, is seen in XCOV18 at $1255.69 \mu \mathrm{Hz}$, within a complex structure of lowamplitude peaks. The fact that this complex structure was not seen in the XCOV13 data may result from a combination of a lower $\mathrm{S} / \mathrm{N}$ ratio and lower frequency resolution.

The $1521 \mu \mathrm{Hz}$ peak seen in XCOV13 and the $1516.26 \mu \mathrm{Hz}$ peak seen during XCOV18 are suggested to belong to the same multiplet (see Sect. 5.2.5).

We note that the peaks at $741.86 \mu \mathrm{Hz}$ and $1280.30 \mu \mathrm{Hz}$, seen only in XCOV13, and $2023.4 \mu \mathrm{Hz}$, seen in all the data, are close to form a linear combination $(741.86 \mu \mathrm{Hz}+$ $1280.30 \mu \mathrm{Hz}=2022.16 \mu \mathrm{Hz}$ ). It is intriguing, and may be meaningful, that this combination was "active" during XCOV13 and "inactive" during XCOV18. While the 2023.4 $\mu \mathrm{Hz}$ frequency must be an independent "parent" mode, it is not possible to know from the WET data alone which of the two other frequencies is the other "parent" mode and which results from their linear combination, since they had the same amplitude during XCOV13 and none was seen during XCOV18. To solve this, we looked back again in the 1989 and 1990 single-site data. We see that the $2023 \mu \mathrm{Hz}$ peak is always present in the data. A peak at $738.72 \mu \mathrm{Hz}$ with an amplitude of $7.2 \mathrm{mma}$ is seen in the October 1990 data. It cannot be the same peak as the one seen at $741.86 \mu \mathrm{Hz}$ during XCOV13 but could be a component of a same multiplet. This peak is not detected in the other runs, unless it could be associated to the peak at $736.67 \mu \mathrm{Hz}$ suspected in the XCOV18 data (see Table 3) and considered as an insignificant linear combination. The $1280 \mu \mathrm{Hz}$ peak is never seen. We conclude that the other parent mode should be the $741.86 \mu \mathrm{Hz}$ one and that the $1280.30 \mu \mathrm{Hz}$ peak may be the linear combination seen only during XCOV13, unless it could be another example of a true resonance. As far as the peaks at $741.86 \mu \mathrm{Hz}, 738.72 \mu \mathrm{Hz}$ and $736.67 \mu \mathrm{Hz}$ are concerned, they could be components of a same multiplet. We come back to theses points later.

We also looked for amplitude variations on shorter time scales by dividing the XCOV18 time series in two halves and comparing the amplitudes of the two corresponding FTs. Restricting ourselves to the independent modes of larger amplitudes, we find some variations for all except one mode: the $1675.61 \mu \mathrm{Hz}$ peak kept a constant amplitude. One finds large variations for the peaks at $1505.54 \mu \mathrm{Hz}$ and $1516.26 \mu \mathrm{Hz}$ which increased by $25 \%$ and $45 \%$ respectively. It is noteworthy to mention that the peak at $938.99 \mu \mathrm{Hz}$, considered as forming a resonance with the $2614 \mu \mathrm{Hz}$ mode and the $1675 \mu \mathrm{Hz}$ mode, shows the largest amplitude change during the two halves of the run $(50 \%)$ while its "companion" modes changed their amplitudes by only $12 \%$ for the $2614 \mu \mathrm{Hz}$ peak and by $0 \%$ for the $1675 \mu \mathrm{Hz}$ peak.
It is finally worthwhile to emphasize once more that the peak at $1675.61 \mu \mathrm{Hz}$ had the same amplitude in XCOV18 and XCOV13, and that its amplitude did not change on the approximately one week time scale separating the two halves of XCOV18. One may wonder whether this mode could be a potentially interesting candidate for a measurement of $\dot{P}$ in HL Tau 76. However, as this frequency might be involved in a resonance with two other modes or a result of their linear combination, any $\dot{P}$ measurement would not be unambiguously interpretable. The value of $\dot{P}$ in those cases could rather be dominated by other mechanisms than the cooling of the degenerate core. Furthermore, we checked that on the longer time scale involved when one takes into account earlier data, the amplitude of this mode is no longer constant. For instance, while it was at $14.8 \mathrm{mma}$ in October 1989 , it increased to $21.8 \mathrm{mma}$ in December 1989 and was back to 15.6 mma in October 1990, to decrease during the two WET campaigns to the same value as observed in October 1989. Furthermore, in the time-resolved spectroscopy performed in October 1997, Kotak et al. (2002b) find that the peak at $1675 \mu \mathrm{Hz}$ was insignificant in the velocity power spectrum. So, our conclusion is that we do not find any mode with a stable enough amplitude whose $\dot{P}$ measurement could unambiguously measure the evolutionary time scale.

\subsection{Fine structure, multiplets}

In this section we look for closely spaced (in frequency) modes with the hope that triplets (or quintuplets) of equal splittings will reveal the $\ell$ values for a number of modes. However, given our confident estimate of the mass and temperature of HL Tau 76, another diagnostic - the period spacings can provide a reliable estimate of $\ell$ as we discuss in the next section.

Recognizing rotationally split modes not only helps in identifying the degree $\ell$ but also leads to an estimate of the rotation period of the star. In the case of HL Tau 76, this exercise is made difficult by the complexity of the power spectrum which does not show clear structures. We nevertheless attempted a mode identification by adopting the following procedure.

We started by looking at closely spaced frequencies in the low frequency part of the amplitude spectrum, in the asymptotic regime where the rotationally split modes should show a uniform frequency separation. However, the only clearly isolated triplet present in the FT which could safely be identified as an $\ell=1$ rotationally split mode is in the "high" frequency part of the FT. It is formed by the peaks 41-43-44 around $1848 \mu \mathrm{Hz}$. For this triplet, the average rotational splitting is $2.55 \mu \mathrm{Hz}$.

Now, we can go back to the low frequency part of the FT and check that any fine structure identified in this part is compatible with similar signatures found at higher frequency, keeping in mind that the modes in the higher frequency part of the FT may not be in the asymptotic regime. In performing this exercise, we start by examining the region between $933.87 \mu \mathrm{Hz}$ and $943.20 \mu \mathrm{Hz}$. It contains 5 significant frequencies but the interpretation depends on whether one considers the $938.99 \mu \mathrm{Hz}$ as a real mode in resonance with the $1675.61 \mu \mathrm{Hz}$ and the 
$2614.55 \mu \mathrm{Hz}$ ones or as a linear combination. In the previous section, we gave arguments in favor of the first hypothesis. In the following discussion, we will consequently consider these three frequencies as corresponding to real modes. One finds then in this group three doublets of comparable frequency separation formed by the peaks $10-11(933.87 \mu \mathrm{Hz}$ and $936.73 \mu \mathrm{Hz}), 11-12(936.73 \mu \mathrm{Hz}$ and $938.99 \mu \mathrm{Hz})$ and $12-14$ $(938.99 \mu \mathrm{Hz}$ and $941.80 \mu \mathrm{Hz})$ separated by $2.86 \mu \mathrm{Hz}, 2.26 \mu \mathrm{Hz}$ and $2.81 \mu \mathrm{Hz}$, respectively, with the peak $15(943.20 \mu \mathrm{Hz})$ $1.4 \mu \mathrm{Hz}$ from peak 14. A possible interpretation is that a $\ell=$ 1 triplet formed by the peaks 11,12 and 14 overlaps with three components formed by the peaks 10,12 and 15 of a $\ell=2$ quintuplet. It is known that in the slow, solid body rotation limit, the pulsation frequencies of a mode of degree $\ell$ and order $k$ for a rotating star, $\sigma_{l, k, m}$, are related to the frequencies in the non rotating case $\sigma_{l, k}$ by:

$\sigma_{l, k, m}=\sigma_{l, k}+m\left(1-C_{l, k}\right) \Omega+o(\Omega)^{2}$

where $C_{l, k}$ takes a simple form in the asymptotic regime: $C_{l, k} \approx$ $1 / \ell(\ell+1)$. We expect the rotational spitting for $\ell=1$ and for $\ell=2$ modes to be in the ratio:

$\delta f_{\ell=1} / \delta f_{\ell=2}=0.6$.

This has been observationally verified in the PG 1159 pulsator PG 1159-035 (Winget et al. 1991).

The average frequency shift in the triplet 11-12-14 is $2.53 \mu \mathrm{Hz}$. As it is almost similar to the value derived for the triplet 41-43-44, we consider that it is due to rotational splitting. The frequency separation within the quintuplet is $5.1 \mu \mathrm{Hz}$ on one side and $4.2 \mu \mathrm{Hz}$ on the other side. The average value, $4.65 \mu \mathrm{Hz}$, compares satisfactorily with the value $(4.22 \mu \mathrm{Hz})$ expected in the asymptotic regime for the rotational splitting of $\ell=2$ modes if the average rotational splitting for the $\ell=1$ modes is $\approx 2.53 \mu \mathrm{Hz}$ in this frequency range. But the ratio $\delta f_{\ell=1} / \delta f_{\ell=2}=0.544$ instead of the expected 0.6 . The peak 12 in this case results from the superposition of the $m=0$ component of the triplet and of one component of the quintuplet, which could be either $m=+1,0$ or -1 .

In the following groups of peaks, one sees four doublets formed by the peaks $16-17(1021.26 \mu \mathrm{Hz}$ and $1024.19 \mu \mathrm{Hz})$, $17-18(1024.19 \mu \mathrm{Hz}$ and $1026.23 \mu \mathrm{Hz}), 18-19(1026.23 \mu \mathrm{Hz}$ and $1029.23 \mu \mathrm{Hz}$ ), separated by $2.93 \mu \mathrm{Hz}, 3.0 \mu \mathrm{Hz}$ and $2.04 \mu \mathrm{Hz}$ and $20-21(1071.58 \mu \mathrm{Hz}$ and $1074.52 \mu \mathrm{Hz})$, separated by $2.94 \mu \mathrm{Hz}$. We consider that the 20-21 doublet is formed by two components of consecutive $m$ values of a $\ell=$ 1 mode split by rotation. Since the doublets $16-17,17-18$ and 18-19 are too close to each other to be consecutive or$\operatorname{der} \ell=1$ modes, we suggest that the group of frequencies 16 to 19 could be formed by a $\ell=1$ triplet overlapping with components of a $\ell=2$ mode. The combination 16-19 could then result from the overlap of an asymmetric triplet formed by the peaks 16-17-18, with a frequency separation of $2.93 \mu \mathrm{Hz}$ on one side and of $2.04 \mu \mathrm{Hz}$ on the other side, with two components of a quintuplet formed by the peaks 17 and 19, separated by $5.04 \mu \mathrm{Hz}$. An alternative combination would be a triplet formed with peaks 17-18-19, also asymmetric with frequency separation of $2.04 \mu \mathrm{Hz}$ on one side and $3.0 \mu \mathrm{Hz}$ on the other side, with two components of a quintuplet formed by peaks 16 and 18 , with a frequency separation of $4.97 \mu \mathrm{Hz}$. In both cases one component of the triplet coincides exactly with one component of the quintuplet. The $m=0$ component of the triplet could be either peak 17 or peak 18 . However, in forming the ratio $\delta f_{\ell=1} / \delta f_{\ell=2}$ in the two options, one finds 0.493 in the first case and 0.507 in the second case, instead of the value 0.6 expected in the asymptotic regime. This weakly favors the second case as a better interpretation. Another argument based on the asymmetry of the triplets, which we will discuss in Sect. 5.4 below, could also favor this second case. However we will conservatively consider the two alternatives as possible solutions in the following discussion. We derive an average rotational splitting of $2.525 \mu \mathrm{Hz}$ for this triplet.

In the high frequency part of the FT, one finds the triplet mentioned earlier composed by the peaks 41-43-44 (1843.48 $\mu \mathrm{Hz}, 1845.74 \mu \mathrm{Hz}$ and $1848.58 \mu \mathrm{Hz})$. One also finds a doublet formed by the peaks $37-39(1670.45 \mu \mathrm{Hz}$ and $1675.61 \mu \mathrm{Hz}$ ). The triplet is slightly asymmetric and the total frequency difference between its two extreme components is $5.10 \mu \mathrm{Hz}$. As the frequency separation between the doublet is $5.16 \mu \mathrm{Hz}$, we interpret this doublet as the two extreme components of a triplet whose the central component is not detected. The peak 38 seen in between the components of the doublet, $0.98 \mu \mathrm{Hz}$ away from peak 39 , cannot be the central component of the triplet. It is a small amplitude peak (4.5 mma) compared to peak 39 (14.6 mma) which is the fifth largest amplitude peak of the FT. The determination of its frequency may have been affected by this large amplitude neighbour. As a consequence, the $m=0$ component of this triplet may not coincide with peak 38 but should be at $\approx 1673.0 \mu \mathrm{Hz}$ (597.7 s). From this triplet and this doublet, we derive an average rotational splitting of $2.56 \mu \mathrm{Hz}$. Finally, one finds a doublet formed by the peaks $48-49(2023.36 \mu \mathrm{Hz}$ and $2027.53 \mu \mathrm{Hz})$, with a frequency separation of $4.17 \mu \mathrm{Hz}$, a value which is difficult to reconcile with the one derived from the doublets and triplets discussed above but in good agreement with the expected rotational splitting for the $\ell=2$ modes. We will argue later about the $\ell=2$ identification for this doublet. The rotational splitting derived for the $\ell=1$ modes from the low and high frequency parts of the FT are in satisfactory agreement. The weak difference between the values derived from the low frequency multiplets $(2.525 \mu \mathrm{Hz})$ and the higher frequency triplets $(2.56 \mu \mathrm{Hz})$ is insignificant given the frequency resolution of our data set. In the following discussion we will use the average value, $2.54 \mu \mathrm{Hz}$, for the rotational splitting of $\ell=1$ modes.

\section{Asteroseismology of HL Tau 76}

\subsection{Period spacing}

Having tentatively identified doublets and triplets as possible $\ell=1$ modes, one looks for a uniform period spacing in their period distribution. For the same reason that the lower frequency multiplets must be closer to the asymptotic regime than the higher frequency ones, we look for periods difference between the low frequency multiplets first. On the assumption that the doublets are two successive components of triplets, we do not 
know which of the components is the $m=0$ mode. Considering first the period differences between the triplet 11-12-14 and the central mode of the next triplet, 17 or 18, we can form two different values for that difference, according to which mode, 17 or 18 , is considered as the $m=0$ in the second triplet: $88.59 \mathrm{~s}$ (corresponding to period of mode 12 - period of mode $17\left(P_{12}-P_{17}\right)$, and $90.53 \mathrm{~s}\left(P_{12}-P_{18}\right)$. The average period difference between these two multiplets is $89.56 \mathrm{~s}$.

Doing the same between the components 17 and 18 of the triplet and the doublet 20-21, one finds four values: $43.2 \mathrm{~s}$ $\left(P_{17}-P_{20}\right), 45.8 \mathrm{~s}\left(P_{17}-P_{21}\right), 41.2 \mathrm{~s}\left(P_{18}-P_{20}\right)$ and $43.8 \mathrm{~s}$ $\left(P_{18}-P_{21}\right)$, with an average period difference of $43.5 \mathrm{~s}$. We note that the $89.56 \mathrm{~s}$ average difference between the first multiplets is $2 \times 44.78 \mathrm{~s}$, a value very close to twice the average period difference between the triplet and the doublet 20-21. We conclude that the period spacing for the $\ell=1$ long period modes should be $\approx 44.1 \mathrm{~s}$ and that there should be a missing multiplet between the triplet $11-12-14$ and the triplet $16-17-18$ or $17-18-19$, centered at a period of $\approx 1020 \mathrm{~s}$.

For the doublet and the triplet at higher frequencies, the period difference between the middle of the peaks 37-39 and the period of the mode 43 is $55.92 \mathrm{~s}$, a value in satisfactory agreement with the value derived at lower frequency, considering that those multiplets may correspond to modes which are not in the asymptotic regime and/or that their period spacing may be affected by mode trapping.

\subsection{Mode identification}

\subsubsection{Search for $\ell=1$ modes}

Adopting an average period spacing deduced from both the low and high frequency multiplets, $\Delta P=48.0 \pm 3.9 \mathrm{~s}$, one can roughly estimate the predicted periods of the $\ell=1$ successive orders, under the assumption of the asymptotic regime.

In the following, we take the period of the mode 43 (the presumably $m=0$ component of this $\ell=1$ triplet) as the reference for the period estimate. The estimated values are shown in Table 5-1. They are listed in the first column. The next three columns give the periods observed during XCOV18, XCOV13 and earlier single-site observations. The last column indicates the relative order difference, $\Delta k$, with the order of the reference mode which is defined as $\Delta k=0$. We should keep in mind that the average period spacing is estimated with a large uncertainty and may formally take any value between $44.2 \mathrm{~s}$ and $52.0 \mathrm{~s}$. The cumulative uncertainty on the predicted period becomes comparable to the period spacing for $\Delta k=48.0 \mathrm{~s} / 3.9 \mathrm{~s} \approx 12$. It means that we cannot predict periods for $\Delta k$ larger than 12 . We add a question mark in Table 5-1 for $\Delta k \geq 12$. However, since with an average period spacing of $48.0 \mathrm{~s}$ one mode is predicted at $1309.8 \mathrm{~s}$ which nicely fit the period of $1308.7 \mathrm{~s}$ observed in archival data (see Sect. 5.2.4) it suggests that the period distribution should not depart much from the one predicted in using this value of the average period spacing up to at least $\Delta k=16$. In cumulating these data, one finds 14 modes, or groups of modes, whose periods may be compared to the expected periods of the $\ell=1$ modes. We find a satisfactory agreement, better or equal to $1 \%$, between the observed and predicted periods for 11 modes. It does not mean that those 11 modes are all $\ell=1$ modes since we have to check whether they could also be explained as well by $\ell=2$ modes, which is discussed in the next section.

It is also possible that the modes whose periods do not fit exactly the distribution predicted by the asymptotic regime for $\ell=1$ are indeed $\ell=1$ modes since the period distribution may not follow exactly the distribution expected in the asymptotic regime. That may be the case for the low order modes. In addition, the periods may be altered by mode trapping, whatever is the hydrogen mass fraction responsible for the trapping. We check a posteriori that the $\ell=1$ mode predicted at $1069.8 \mathrm{~s}$ is quite close to the observed $1064.97 \mathrm{~s}$ one $(939 \mu \mathrm{Hz})$ which we suspected to be a mode in resonance with the $1675 \mu \mathrm{Hz}$ and the $2614 \mu \mathrm{Hz}$ modes rather than a linear combination. This reinforces our identification of this frequency as a real mode and increases the number of independent modes to 34 . It must be noticed that among the 22 expected $\ell=1$ modes in the period range $\approx 380-1400 \mathrm{~s}$, we detected only 11 potential $\ell=1$ modes in the cumulative data.

\subsubsection{Search for $\ell=2$ modes}

The next step consists in using what has been derived about the period spacing and the rotational splitting for the $\ell=1$ modes to predict the period distribution of the possible $\ell=2$ modes. The rotational splitting of $\ell=1$ and $\ell=2$ modes are in the ratio 0.6 in the asymptotic regime. The period spacing for a series of modes of degree $\ell$, given by (Tassoul 1980)

$$
\Delta P_{\ell}=\Pi_{0} /[\ell(\ell+1)]^{1 / 2}
$$

where $\Pi_{0}$ is related to the structure of the star, implies that the ratio of the period spacings

$\Delta P_{\ell=2} / \Delta P_{\ell=1}=1 / \sqrt{ } 3$

Since the average period spacing is $48.0 \pm 3.9 \mathrm{~s}$ and the average rotational splitting is $2.54 \mu \mathrm{Hz}$ for the $\ell=1$ modes, the corresponding values for the $\ell=2$ modes should be $27.7 \pm 2.2 \mathrm{~s}$ and $4.23 \mu \mathrm{Hz}$. We also need to identify at least one $\ell=2$ mode to be used as a reference value for the period distribution.

The average period spacing for the $\ell=1$ modes derived in the previous section, $\Delta P=48.0 \mathrm{~s}$, is quite consistent with the average spacing of $44.98 \mathrm{~s}$ in one of the models with a thick hydrogen layer by Brassard et al. (1992) whose parameters are close enough to HL Tau 76 (see for instance their model 60204). Very similar values are found by Corsico et al. (2002). Furthermore, in considering the periods of the modes in many of the models computed by Brassard et al. (1992) as well as in those computed by Bradley (1993) and Corsico et al., one finds many examples of $\ell=1$ and $\ell=2$ modes which overlap in frequency. For instance, in the Brassard et al. (1992) model 60204, there are 6 such coincidences of modes $\ell=1$ and $\ell=2$ differing in frequency by less than $11 \mu \mathrm{Hz}$, within the frequency range $1100-4300 \mu \mathrm{Hz}$. In examining the frequency list in Table 3 one finds regions with series of close frequency peaks which cannot be explained as $\ell=1$ triplets 
Table 5. 1. Search for $\ell=1$ modes in HL Tau 76 with $\Delta P_{\ell=1}=48.0 \mathrm{~s}$.

\begin{tabular}{|c|c|c|c|c|}
\hline $\begin{array}{l}\text { Predicted } \\
\text { Period (s) }\end{array}$ & $\begin{array}{c}\text { Observed periods (s) } \\
\text { (XCOV18) }\end{array}$ & $\begin{array}{c}\text { Observed periods (s) } \\
\text { (XCOV13) }\end{array}$ & $\begin{array}{c}\text { Observed periods (s) } \\
\text { Archival data }\end{array}$ & $\Delta k$ \\
\hline 1405.8 & 1390.8 & & & $+18 ?$ \\
\hline 1357.8 & & 1347.9 & 1353.7 & $+17 ?$ \\
\hline 1309.8 & & & 1308.7 & $+16 ?$ \\
\hline 1261.8 & & & & +15 ? \\
\hline 1213.8 & & & & +14 ? \\
\hline 1165.8 & & & & $+13 ?$ \\
\hline 1117.8 & & & & +12 ? \\
\hline 1069.8 & $1070.8,1067.5,1065.0$ & 1064.5 & & +11 \\
\hline 1021.8 & & & & +10 \\
\hline 973.8 & $979.2-971.6$ & & & +9 \\
\hline 925.8 & $933.2,930.6$ & 932.5 & & +8 \\
\hline 877.8 & & & & +7 \\
\hline 829.8 & & & & +6 \\
\hline 781.8 & $799.1-792.7$ & $796.5,781.0$ & & +5 \\
\hline 733.8 & & & 748.5 & +4 \\
\hline 685.8 & & & 689.3 & +3 \\
\hline 637.8 & 659.5 & 657.4 & 628 & +2 \\
\hline 589.8 & $598.6,597.1,596.8$ & 597.0 & & +1 \\
\hline 541.8 & $542.4,541.8,540.9$ & 541.0 & & 0 \\
\hline 493.8 & $493.2,494.2$ & & 494 & -1 \\
\hline 445.8 & & 449.8 & & -2 \\
\hline 397.8 & 382.5 & 382.5 & & -3 \\
\hline
\end{tabular}

nor by $\ell=2$ quintuplets alone. We suggest that they should result from such multiplets overlapping. If we exclude the presence of $\ell=3$ modes on visibility arguments, one is left with this only explanation to interpret the 5 peaks seen between 933 and $943 \mu \mathrm{Hz}$, the 5 close frequencies detected between 1251 and $1261 \mu \mathrm{Hz}$, as well as the series of frequencies detected between 1505 and $1516 \mu \mathrm{Hz}$.

As there is no complete quintuplet identified, we do not have a solid reference $\ell=2, m=0$ mode to start with to predict the $\ell=2$ modes period distribution. Any suspected $\ell=2$ mode in our data is a component of an incomplete multiplet of unknown $m$ value. In the search for fine structures in the frequency spectrum, it was implicitely assumed that the rotational splitting does not change within the range of the periods observed in HL Tau 76. That is consistent with the hypothesis that the rotation law in HL Tau 76 must be close to uniform rotation. In this case, since the frequency shift induced by the rotational splitting is fixed for a given $\ell$, the uncertainty on the period scale is smaller if we can identify a high frequency peak as a $\ell=2$ mode. Fortunately, we have such a possible $\ell=2$ candidate with the high amplitude doublet at 2023.36 and $2027.53 \mu \mathrm{Hz}$. This identification is based on the frequency separation of these two peaks, $4.17 \mu \mathrm{Hz}$, which is in good agreement with the expected rotational splitting for $\ell=2$ modes. Considering this doublet as two components of an $\ell=2$ mode and the adopted rotational splitting, there are
4 possible values for the period of the $m=0$ component of the quintuplet: $495.3 \mathrm{~s}, 494.2 \mathrm{~s}, 493.1 \mathrm{~s}$ and 492.1 . So the central mode period of this incomplete multiplet is known within a $3.2 \mathrm{~s}$ interval. The center of this interval is at $493.7 \mathrm{~s}$ while the high amplitude component (peak 48 in Table 3) is at $494.2 \mathrm{~s}$. We consider the mode 48 as a good enough reference point for the period distribution of the $\ell=2$ modes. In addition, it is the second highest amplitude mode, so its period is quite precisely determined.

Table 5-2 shows the corresponding period distribution for $\ell=2$ modes, assuming the asymptotic regime, a period spacing of $27.7 \mathrm{~s}$ and the period of mode 48 as the reference value. The first column shows the predicted periods. The following three columns list the periods present in XCOV18, XCOV13 and earlier single-site observations, respectively. The last column indicates again the relative order difference with the reference mode, which is $\Delta k=0$. Regions of the spectrum where none of the observed periods fits close enough with any predicted values are not listed. Since the average period spacing is known with a statistical error of $2.2 \mathrm{~s}$ for the $\ell=2$ modes, we cannot formally predict the periods and the relative $\Delta k$ for $\Delta k \geq 27.7 \mathrm{~s} / 2.2 \mathrm{~s}=12-13$, since the uncertainty on the period becomes comparable to the period spacing. For this reason all predicted periods larger than $800 \mathrm{~s}(\Delta k \geq 12)$ are followed with a question mark. However, since we argued in the previous section that the true period distribution for the $\ell=1$ modes should 
Table 5. 2. Search for $\ell=2$ modes in HL Tau 76 with $\Delta P_{\ell=2}=27.7 \mathrm{~s}$.

\begin{tabular}{|c|c|c|c|c|}
\hline $\begin{array}{l}\text { Predicted } \\
\text { Period (s) }\end{array}$ & $\begin{array}{c}\text { Observed periods (s) } \\
(\mathrm{XCOV} 18)\end{array}$ & $\begin{array}{c}\text { Observed periods (s) } \\
\text { (XCOV13) }\end{array}$ & $\begin{array}{c}\text { Observed periods (s) } \\
\text { Archival data }\end{array}$ & $\Delta k$ \\
\hline 1380.6 & 1390.8 & & & +32 ? \\
\hline 1352.9 & & 1347.9 & 1353.7 & +31 ? \\
\hline . & . & . & . & . \\
\hline . & . & . & . & . \\
\hline 1075.9 & $1060.2-1070.8$ & 1064.5 & & $+21 ?$ \\
\hline . & & . & . & . \\
\hline . & & . & . & . \\
\hline 965.1 & $979.2-971.6$ & & & $+17 ?$ \\
\hline 937.4 & $930.6-933.2$ & 932.5 & & $+16 ?$ \\
\hline . & . & . & . & . \\
\hline . & 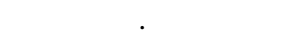 & . & . & . \\
\hline 798.9 & $792.7-799.1$ & $796.5,781.0$ & & +11 \\
\hline . & . & . & . & . \\
\hline . & . & . & . & . \\
\hline 743.5 & & & 748.5 & +9 \\
\hline 688.1 & & & 689.3 & +7 \\
\hline 660.4 & $659.5-664.2$ & & 665 & +6 \\
\hline 632.7 & & & 628 & +5 \\
\hline 605.0 & $596.8,597.1,598.0$ & 597.0 & & +4 \\
\hline 577.3 & & & & +3 \\
\hline 549.6 & $540.9,541.7,542.4$ & 541.0 & & +2 \\
\hline 521.9 & & & & +1 \\
\hline 494.2 & $493.2,494.2$ & 494.2 & 494 & +0 \\
\hline 466.5 & & & & -1 \\
\hline 438.8 & & 449.8 & & -2 \\
\hline 411.1 & & & & -3 \\
\hline 383.4 & 382.4 & 382.5 & & -4 \\
\hline
\end{tabular}

not depart much from the one predicted with the average period spacing of $48.0 \mathrm{~s}$, this should be true also for the period distribution of the $\ell=2$ modes, if the reference period is a true $\ell=2$ mode. We also point out that the predicted $\ell=2$ mode with period $1380.6 \mathrm{~s}$ is close to the observed one at $1390.8 \mathrm{~s}$, which should be $\Delta k=32$. It suggests that the period distribution for the $\ell=2$ modes does not depart much from the one predicted with the average period spacing of $27.7 \mathrm{~s}$ even up to $\Delta k=32$.

Again comparing the observed periods with the predicted periods, one sees that among the 15 groups of observed periods, 12 fit satisfactorily with $\ell=2$ modes, with less than $1 \%$ difference in period.

From Tables 5-1 and 5-2, one finds that at least 9 modes or groups of modes have periods within $1 \%$ of the period of either an $\ell=1$ or an $\ell=2$ mode. They very probably result from the overlap between $\ell=1$ and $\ell=2$ modes.

Comparing the periods detected during the two WET campaigns, one finds three modes which were seen during XCOV13 and are not present during XCOV18. Since the arguments used to estimate the periods of the modes are based on the period spacing deduced from the XCOV18 data alone, it is worth checking whether and how precisely these additional modes fit the proposed identification. They are the periods $1347.9 \mathrm{~s}(741.86 \mu \mathrm{Hz}), 781.0 \mathrm{~s}(1280 \mu \mathrm{Hz})$ and $449.8 \mathrm{~s}$ $(2223 \mu \mathrm{Hz})$.

The first one at $1347.9 \mathrm{~s}(741.86 \mu \mathrm{Hz})$ fits satisfactory, within $5 \mathrm{~s}$, the period of the predicted $\ell=2$ mode at $1352.9 \mathrm{~s}$ $(\Delta k=+31$ in Table $5-2)$. But it is also at $10 \mathrm{~s}$ of the predicted mode $\ell=1$ at $1357.8 \mathrm{~s}(\Delta k=+17$ in Table 5-1).

We already argued that the second one at $781.0 \mathrm{~s}$ $(1280 \mu \mathrm{Hz})$ probably results from the linear combination of the $741.86 \mu \mathrm{Hz}$ mode with the $2023 \mu \mathrm{Hz}$ one. We consequently would not consider it as an independent mode unless there is one predicted mode at this period. That is precisely the case since there is a predicted mode at $781.8 \mathrm{~s}$ in the $\ell=1$ series (Table 5-1). It suggests that this period could correspond to a real mode and points to the possibility that it is another example of a true resonance between the three modes. The third one has a period of $449.8 \mathrm{~s}(2223 \mu \mathrm{Hz})$. The closest predicted period is a $\ell=1$ mode (Table $5-1$ ) at $445.8 \mathrm{~s}$. So, the three additional modes present in XCOV13 and not seen in XCOV18 fit 
satisfactorily with the periods predicted by the period spacing deduced from XCOV18 data.

\subsubsection{Back to the XCOV18 amplitude spectrum}

We used the asymptotic period distribution to predict the periods of the expected $\ell=1$ and $\ell=2$ modes. It is worth reexamining the best amplitude spectrum obtained for HL Tau 76, from the XCOV18 WET campaign, to check whether we could identify some additional modes not selected by our significance criterion, but whose periods are close enough to the predicted ones to suggest that they could be real modes. In that case, they would not have been selected because their amplitudes were below, or close to the adopted detection limit during XCOV18. We also check whether those modes were detected in previous observations.

Visual inspection of the XCOV18 amplitude spectrum (Figs. 2 and 3) reveals 2 such peaks. The first one at $1353.7 \mu \mathrm{Hz}$ (738.7 s) has a low amplitude of $\approx 2.6 \mathrm{mma}$. This peak is quite close to the one found in the early observations of Warner \& Nather $(1970,1972)$ as reanalysed by Page (1972) at $1336.0 \mu \mathrm{Hz}(748.5 \mathrm{~s})$. We note that there is one $\ell=2$ mode predicted at $743.5 \mathrm{~s}$ (Table 5-2). Taken at face value as the $m=0$ component of a $\ell=2$ quintuplet, and using the rotational splitting of $4.23 \mu \mathrm{Hz}$ for $\ell=2$ modes, that would predict the $m=-2$ component at $1336.5 \mu \mathrm{Hz}$ and the $m=+2$ component at $1353.4 \mu \mathrm{Hz}$. We consider this excellent agreement with the observed frequencies as strong evidence that the suspected peak at $1353.7 \mu \mathrm{Hz}$ is real and corresponds to a component of an $\ell=2$ mode whose other component at $1336.0 \mu \mathrm{Hz}$ happened to be the largest amplitude mode in the early observations of HL Tau 76.

The other one is at $2536.06 \mu \mathrm{Hz}(394.3 \mathrm{~s})$ and has an amplitude of 2.9 mma. It has not been observed before. The closest observed frequency is the $2614 \mu \mathrm{Hz}$ (382 s) one of large amplitude seen in all the observing seasons. This small amplitude peak is close to the predicted $\ell=1$ mode at $397.8 \mathrm{~s}$ (Table 5-1) and may possibly be identified with that mode.

In the following, we will keep both the $738.7 \mathrm{~s}$ and the 394.3 s periods as real modes according to the above mentioned argument. However, we will keep in mind that they are below the detection limit in XCOV18 by marking them with a colon (:) in the identification list proposed below (Table 6).

\subsubsection{Back to archival data}

The same checking operation can be performed with the earlier data. Looking back in the early observations of HL Tau 76, we already mentioned that among the dominant periods at $748.5 \mathrm{~s}$, 665 s, 628 s and 494 s (Warner \& Nather 1970, 1972; Page 1972; Fitch 1973) only two were seen at a significant level in the XCOV13 and XCOV18 data, the ones at $665 \mathrm{~s}$ and at $494 \mathrm{~s}$. It is interesting to check whether the two missing ones can be predicted by the asymptotic extrapolation at their correct values. We have shown in the previous section that the mode at $748.5 \mathrm{~s}$, which had the largest amplitude in the original discovery data, may be associated with the $738.7 \mathrm{~s}$ which is present in the XCOV18 data below detection limit. They are both probable components of the $\ell=2$ mode predicted at $743.5 \mathrm{~s}(\Delta k=+9$ in Table 5-2). For the mode at $628 \mathrm{~s}$, the closest $\ell=1$ mode would be at $637.8 \mathrm{~s}(\Delta k=+2$ in Table 51) while the closest $\ell=2$ mode would be at $632.7 \mathrm{~s}(\Delta k=+5$ in Table 5-2). This suggests that this mode is also better fit as an $\ell=2$ mode.

In the data obtained in 1989 and 1990, we also find a few significant peaks in their respective amplitude spectra which are not seen at significant levels in XCOV13 or in XCOV18. In the October 1990 data, we find a peak at $738.72 \mu \mathrm{Hz}(1353.7 \mathrm{~s}$ ) with an amplitude of $7.2 \mathrm{mma}$. This frequency is quite close to the $736.67 \mu \mathrm{Hz}$ (1357.5 s) listed in Table 3 after the first step of the selection process and rejected as insignificant after the second step. It was anyway considered as a linear combination. However, considering that there is an $\ell=1$ mode predicted at $1357.8 \mathrm{~s}(736.5 \mu \mathrm{Hz})$ and an $\ell=2$ mode predicted at $1352.9 \mathrm{~s}$ it is quite possible that it corresponds to a real mode rather than to a linear combination. A $764.1 \mu \mathrm{Hz}$ (1308.7 s) was seen in the December 1989 data with a small amplitude (3.6 mma). It could be a linear combination between the $2614.5 \mu \mathrm{Hz}$ and the $1847.7 \mu \mathrm{Hz}$ modes seen in the same data (they correspond to the $2614.55 \mu \mathrm{Hz}$ and $1848.58 \mu \mathrm{Hz}$ seen in XCOV18). There is also in this case an $\ell=1$ mode predicted at $1309.8 \mathrm{~s}$. This frequency could consequently be a linear combination as well as a real mode. Finally, in the November 1989 data, we find a peak at $1450.8 \mu \mathrm{Hz}(689.3 \mathrm{~s})$ with an amplitude of 8.2 mma. Examination of Tables 5-1 and 5-2 shows that there is an $\ell=1$ mode predicted at $685.8 \mathrm{~s}$ and an $\ell=2$ mode predicted at $688.1 \mathrm{~s}$. It suggests that this frequency may be identified with the $\ell=2$ mode. These three periods present in archival single-site data are included in Tables 5-1 and 5-2. However, for the final identification we conservatively exclude the two ambiguous cases which could be either a real mode or a linear combination but keep the mode at $689.3 \mathrm{~s}$.

\subsubsection{Conclusions}

To summarize: from XCOV18, we find 36 independent modes. This includes the mode at $939 \mu \mathrm{Hz}$, considered as a mode in resonance with two other modes, and the two modes at $1353.7 \mu \mathrm{Hz}$ and $2536.06 \mu \mathrm{Hz}$ because the first one coincides with a period observed in the early data and the second one coincides with a mode predicted by the asymptotic period distribution. XCOV13 provides 11 modes of which 4 are not present in XCOV18. Among these four, we discussed the case of the two modes at $742 \mu \mathrm{Hz}$ and $1280 \mu \mathrm{Hz}$ which form a linear combination with a third independent mode at $2023 \mu \mathrm{Hz}$. We showed that they probably form a resonance and are real modes. For this reason we add these four modes as independent modes from XCOV13. Finally, the archival data provide 5 modes, among which only 2 are present in XCOV 18 and/or XCOV13, which adds another 3 independent modes. The total number of independent modes found in the cumulative data sets is 43 . 
In comparing the periods observed with the ones predicted by the period spacing and taking into account the rotational splitting, we make a preliminary identification of the modes. From Tables 5-1 and 5-2, which list the predicted periods for $\ell=1$ and $\ell=2$ modes, one sees a number of cases were the observed periods could be identified as either $\ell=1$ or as $\ell=2$ modes.

For some of those cases, we offered a tentative identification above, which lifts the degeneracy between the $\ell=1$ and $\ell=2$ identification. On the basis of the period differences between the observed values and the expected values from the asymptotic relation and from the rotational splitting, we can choose one solution rather than another one. The preliminary identification of the modes observed in HL Tau 76 is listed in Table 6. We discuss some remaining difficult or ambiguous cases below.

The five modes between $799.1 \mathrm{~s}$ and $792.7 \mathrm{~s}$ could correspond to the $\ell=2$ mode predicted at $798.9 \mathrm{~s}$. However, their frequency separation does not fit the expected rotational splitting. They all show a small amplitude $(\leq 5.2 \mathrm{mma})$ during XCOV18 so that their frequencies may not be very precisely determined. The closest predicted $\ell=1$ mode is at $781.8 \mathrm{~s}$ and is seen in the XCOV13 data (at 781 s). Could this group of periods results from the overlap of an $\ell=1$ and an $\ell=2$ multiplet? Considering the value of the rotational splitting associated to $\ell=1$ modes, ones does not expect a component of the $\ell=1$ triplet at a period longer than $784 \mathrm{~s}$, which is the extreme case if the observed $781.0 \mathrm{~s}$ is the prograde $(m=+1)$ component of the triplet. The multiplets should not overlap within the period range covered by this group. Considering the predicted mode at $798.9 \mathrm{~s}$ at face value as an $\ell=2, m=0$ mode and applying the rotational splitting for $\ell=2$, one predicts the periods of the other components of the quintuplet at $804.3 \mathrm{~s}$, $801.6 \mathrm{~s}, 796.2 \mathrm{~s}$ and $793.5 \mathrm{~s}$ for $m=-2,-1,+1$ and +2 respectively. We note that $793.5 \mathrm{~s}$ falls between the observed periods at $792.7 \mathrm{~s}$ and $794.1 \mathrm{~s}$ and that $798.9 \mathrm{~s}$ falls between the observed periods at $798.3 \mathrm{~s}$ and $799.1 \mathrm{~s}$. It suggests that both double-peaks at 792.7-794.1 s and 798.3-799.1 s could in fact be single peaks incorrectly resolved by the extraction algorithm because of their low amplitudes and correspond to the two components $m=+2$ and 0 of the $\ell=2$ mode of which the $m=+1$ component corresponds to the $796.4 \mathrm{~s}$ period. The other two components of the quintuplet are missing. For these cases, we conservatively list all the periods in Table 6 but identify the two double-peaks as single modes.

The group of modes 29 to 36 , from $1505.5 \mu \mathrm{Hz}$ to $1516 \mu \mathrm{Hz}$, forms the next complex region in the FT as shown in Table 3 . As there are exactly 8 peaks in the FT of XCOV 18 in this frequency range, it suggests that they could correspond to the superposition of a complete triplet and a complete quintuplet. However, we remark that Dolez \& Kleinman (1997) found a peak at $1520.96 \mu \mathrm{Hz}$ during XCOV13 while a peak at $1516.26 \mu \mathrm{Hz}$ (36 in Table 3) was present during XCOV18. They cannot be the same peak because their frequency difference, $4.7 \mu \mathrm{Hz}$, is 7 and 4 times the frequency resolution of the WET run where they were detected and both are large amplitude peaks (10.7 mma and $10.3 \mathrm{mma}$ respectively), i.e. their frequencies are determined with a much better accuracy
Table 6. Proposed Identification of the modes in HL Tau 76.

\begin{tabular}{|c|c|c|c|}
\hline $\begin{array}{l}\text { Periods (s) } \\
(\mathrm{XCOV} 18)\end{array}$ & $\begin{array}{l}\text { Period (s) } \\
\text { (XCOV13) }\end{array}$ & $\begin{array}{l}\text { Period (s) } \\
\text { Archives }\end{array}$ & $\ell$ \\
\hline \multirow[t]{3}{*}{1390.8} & & & 2 \\
\hline & 1347.9 & 1353.7 & 2 \\
\hline & & 1308.7 & 1 \\
\hline 1070.8 & & & 2 \\
\hline 1067.5 & & & 1 \\
\hline 1065.0 & 1065.0 & & 1,2 \\
\hline 1061.8 & & & 1 \\
\hline 1060.2 & & & 2 \\
\hline 979.2 & & & 1,2 \\
\hline 976.4 & & & 1,2 \\
\hline 974.4 & & & 1,2 \\
\hline 971.6 & & & 1,2 \\
\hline 933.2 & 932.5 & & 1 \\
\hline 930.6 & & & 1 \\
\hline 799.1 & & & 2 \\
\hline 798.3 & & & 2 \\
\hline 796.4 & 796.5 & & 2 \\
\hline 794.1 & & & 2 \\
\hline \multirow[t]{3}{*}{792.7} & & & 2 \\
\hline & 781.0 & & 1 \\
\hline & & 748.5 & 2 \\
\hline \multirow[t]{2}{*}{ 738.7: } & & & 2 \\
\hline & & 689.3 & 2 \\
\hline 664.2 & & 665.0 & 2 \\
\hline 663.6 & & & $?$ \\
\hline 662.8 & & & $?$ \\
\hline 662.3 & & & 2 \\
\hline 661.9 & & & $?$ \\
\hline 661.4 & & & 2 \\
\hline 660.1 & & & $?$ \\
\hline \multirow[t]{3}{*}{659.5} & & & 2 \\
\hline & 657.4 & & 2 \\
\hline & & 628.0 & 2 \\
\hline 598.6 & & & 1 \\
\hline 597.1 & 597.0 & & 1 \\
\hline 596.8 & & & 1 \\
\hline 542.4 & & & 1 \\
\hline 541.8 & & & 1 \\
\hline 540.9 & 541.0 & & 1 \\
\hline 494.2 & 494.2 & 494.0 & 2 \\
\hline \multirow[t]{2}{*}{493.2} & & & 2 \\
\hline & 449.8 & & 1 \\
\hline 394.3: & & & 1 \\
\hline 382.5 & 382.5 & & 2 \\
\hline
\end{tabular}


than the formal frequency resolution. Such a difference suggests that they could be two consecutive components of an $\ell=2$ quintuplet. In this case, we get 9 frequencies in this frequency interval that cannot be fit by the overlap of a triplet and a quintuplet. However, one may question the reality of mode 31 (at $1508.71 \mu \mathrm{Hz}$ ) whose amplitude $(3.49 \mathrm{mma}$ ) is small compared to its close neighbours with $11.90 \mathrm{mma}$ amplitude for peak 30 at $1.88 \mu \mathrm{Hz}$ and with 10.84 mma amplitude for peak 32 at $1.12 \mu \mathrm{Hz}$. It is not certain again that our extraction algorithm can disantangle such a small amplitude peak from such a complex and crowded frequency region. But, even if one rejects the peak 31 as insignificant, one is left with a difficult identification problem for the remaining 8 peaks. Four or five of them could be the components of an $\ell=2$ mode. That could be either the peaks $30(1506.83 \mu \mathrm{Hz}), 34(1511.96 \mu \mathrm{Hz}), 36(1516.26 \mu \mathrm{Hz})$ and the $1520.96 \mu \mathrm{Hz}$ peak seen in XCOV13, separated by 5.1 , 4.3 and $4.7 \mu \mathrm{Hz}$ respectively or the peaks $29(1505.54 \mu \mathrm{Hz})$, $32(1509.83 \mu \mathrm{Hz}), 34(1511.96 \mu \mathrm{Hz}), 36(1516.26 \mu \mathrm{Hz})$ and the $1520.96 \mu \mathrm{Hz}$ of XCOV13, separated by $4.3,2.1,4.3$ and $4.7 \mu \mathrm{Hz}$ respectively. In the last case one would have a complete quintuplet, rather asymmetrical, with the central component at $1511.96 \mu \mathrm{Hz}$ which fits almost perfectly the predicted $\ell=2$ mode at $660.4 \mathrm{~s}$. We give a preliminary identification of $\ell=2$ for those five modes in Table 6. However, there is no $\ell=1$ mode predicted in this period range able to overlap with the quintuplet. The closest $\ell=1$ modes are at $1458.1 \mu \mathrm{Hz}$ and $1567.9 \mu \mathrm{Hz}$ far from the frequency range covered by this group of modes, even if one takes into account the rotational splitting. The assumption of an overlap between $\ell=1$ and $\ell=2$ modes does not work for this group, at least in the limit of the assumptions made in this paper that the period distribution follows the asymptotic regime. This tentative identification leaves at least 3 modes unexplained which are not small amplitude peaks (12 mma, $7.6 \mathrm{mma}$ and $7.3 \mathrm{mma}$ ). They are left with a question mark in Table 6 . It should be pointed out that large amplitude variations were observed during XCOV18 in this region (Sect. 4.1). The peaks at $1505.54 \mu \mathrm{Hz}$ (29) and at $1516.26 \mu \mathrm{Hz}$ (36) showed the largest amplitude variations. These variations may explain why we find one close companion to each of these large amplitude peaks (30 and 35). That may be the reason why this frequency domain cannot be satisfactorily interpreted. The two modes below the detection limit of XCOV18 added according to the discussion presented in 5.2.3 are marked with a colon (:).

Kotak et al. (2002b), in their analysis of time-resolved spectroscopy of HL Tau 76, suggest that most of the modes for which they detect a significant peak in the velocity power spectrum are $\ell=1$ modes (for the periods $657 \mathrm{~s}, 597 \mathrm{~s}, 541 \mathrm{~s}, 494 \mathrm{~s}$ and $382 \mathrm{~s}$ ) while the last one at $781 \mathrm{~s}$ would be better identified as an $\ell=2$ mode. Their proposed identification relies on the value of the parameter $R_{v}$, the relative velocity to flux amplitude ratio, after van Kerkwijk et al. (2000). Their identifications are consistent with the values of $R_{v}$ that they find. Our identifications for the 6 modes we have in common agree for the two modes at $597 \mathrm{~s}$ and $541 \mathrm{~s}$, which we also identify as $\ell=1$ modes, but disagree for the remaining modes. The modes at $657.4 \mathrm{~s}, 494.2 \mathrm{~s}$ and $382.5 \mathrm{~s}$, which we rather identify as $\ell=2$ modes, should have been detected with a larger relative velocity to flux amplitude ratio than the values they report. We do not offer presently any explanation for this disagreement.

Finally, it is worth comparing the periods found in HL Tau 76 with those of G 29-38 (Kleinman et al. 1998). There are both some similarities and differences. The periods observed in G 29-38 covers also a large range from $110 \mathrm{~s}$ to $1240 \mathrm{~s}$, comparable to the range observed in HL Tau 76, from $380 \mathrm{~s}$ to $1390 \mathrm{~s}$, but shifted towards shorter periods. Since G 29-38 is both hotter and more massive than HL Tau 76 (11820 K and $0.69 M_{\odot}$, according to Bergeron et al. 2004), such a global shift to shorter periods is expected. The main difference is that while all the periods seen in G 29-38 may be attributed to $\ell=1$ modes, HL Tau 76 exhibits a more complex mixture of $\ell=1$ and $\ell=2$ modes, with some overlapping between both as discussed above. Among the modes identified as $\ell=1$ in HL Tau 76, we find many similarities with the periods found in G 29-38: for instance the period at $933 \mathrm{~s}$, 781 s, 597 s, $542 \mathrm{~s}$ and $394 \mathrm{~s}$ in HL Tau 76 are close to the periods at $915 \mathrm{~s}, 771 \mathrm{~s}, 610 \mathrm{~s}, 552 \mathrm{~s}$ and $400 \mathrm{~s}$, respectively, in $\mathrm{G} 29-38$. But all the other similar periods do not correspond to similar $\ell$ values in both stars.

\subsection{Rotation rate}

From the multiplets identified in HL Tau 76 we can derive the average rotation period of the star. In averaging the frequency separation of the doublets and the triplets, all considered as $\ell=1$ modes split by rotation, we have obtained an average rotational splitting $\delta f=2.54 \mu \mathrm{Hz}$. The average rotation period derived from the rotational splitting is 2.2 days. This rotational period for HL Tau 76 is in the range of rotation periods derived from asteroseismology for other pulsating white dwarfs. It confirms that low and intermediate mass stars lose most of their angular momentum during their evolution from the main sequence to the white dwarfs. There is no significant signature of a dependence of the rotational splitting on the period, which is compatible with a uniform rotation but does not prove it (Kawaler et al. 1999). However, since there are quite few rotationally split modes detected in the data analysed here, we cannot exclude a more complex rotation law.

\subsection{Search for weak magnetic field}

The fine structure of the Fourier amplitude spectrum reveals only three probable complete triplets and no quintuplets. The triplets are formed by the peaks $41-43-44,16-17-18$ or 17-18-19 and 11-12-14, in the order of increasing period. These triplets are interpreted as $\ell=1$ modes split by rotation. Rotation by itself would produce symmetric triplets in the limit of slow rotation. However none of the observed triplets is symmetrical. The asymmetry could result from second order rotation effects or from magnetic field. With an average rotation period of 2.2 days, we do not expect the second order effects of rotation to have any detectable consequence on rotational splitting. The influence of weak magnetic fields on the $g$-modes in white dwarfs has been analysed by Jones et al. (1989). Their 
effect is to shift the frequencies to higher values proportionally to $m^{2} B^{2}$ and the frequency shifts increase with the order of the mode, i.e. with the period. This produces an asymmetry in the triplets, $\Delta f_{m}=\left[\left(f_{m=+1}-f_{m=0}\right)-\left(f_{m=0}-f_{m=-1}\right)\right] / 2$, the retrograde component $m=-1$ being closer to the central component $m=0$ than the prograde component $m=+1$. The two triplets $41-43-44$ and $11-12-14$ are very similar in their asymmetry with $f_{m=0}-f_{m=-1}=2.26 \mu \mathrm{Hz}$ in both cases and $f_{m=+1}-f_{m=0}=2.84 \mu \mathrm{Hz}$ and $2.81 \mu \mathrm{Hz}$ respectively. In the case of the third triplet, we discussed the two plausible solutions where the triplet could be formed by the peaks $16-17-18$ or $17-18-19$. If the triplet is formed by the peak $16-17-18$, the observed asymmetry cannot be due to the effect of magnetic field since $f_{m=0}-f_{m=-1}=2.93 \mu \mathrm{Hz}$ and $f_{m=+1}-f_{m=0}=2.04 \mu \mathrm{Hz}$, i.e. the $m= \pm 1$ would be shifted in the wrong direction. If the triplet is $17-18-19$ we find $f_{m=0}-f_{m=-1}=2.04 \mu \mathrm{Hz}$ and $f_{m=+1}-f_{m=0}=3.0 \mu \mathrm{Hz}$. If we assume that the asymmetry in the triplets is due to a weak magnetic field, this rules out the solution 16-17-18 for the third triplet. The asymmetry $\left(\Delta f_{m}=0.29 \mu \mathrm{Hz}\right)$ observed in the best identified triplet (41-43-44) centered at the period of $541 \mathrm{~s}$ could be explained with a weak dipole field of $\approx 1-2 \times 10^{3} \mathrm{G}$, from the results of Jones et al. (their Fig. 1). However, when we consider the other two triplets we do not find the expected variation of the frequency shift with increasing period: $\Delta f_{m}=0.48 \mu \mathrm{Hz}$ at $974 \mathrm{~s}$ for the triplet $17-18-19$ but $\Delta f_{m}=0.28 \mu \mathrm{Hz}$ at $1065 \mathrm{~s}$ for the triplet $11-12-14$. If there is a hint for the presence of a weak magnetic field in HL Tau 76, as inferred from the asymmetrical triplets, there is no conclusive evidence. Since there is no complete $\ell=2$ quintuplets, it is not possible to check the effect of the $m^{2}$ dependence on the frequency shift.

\subsection{Mass of the $H$ outer layers}

The average period spacing for $\ell=1$ modes derived from the present study is $\Delta P=48.0 \mathrm{~s}$. This value translates to $\Pi_{0}=67.9 \mathrm{~s}$. A detailed comparison of the periods of observed $\ell=1$ and $\ell=2$ modes with the periods of modes calculated in a grid of models will be presented in a forthcoming paper. It should provide the model or series of models best fitting the observed periods. With a total of $\approx 40$ observed modes, the model should be rather well constrained if the pulsations of HL Tau 76 are still well described by linear nonradial pulsation theory. As HL Tau 76 shows this large number of modes on an extended range of periods, from $382 \mathrm{~s}$ to $1390 \mathrm{~s}$, there are very few modes of consecutive orders for a given degree. Due to this unfortunate circumstance, one cannot build the period spacing-Period ( $\Delta P$-vs. $-P$ ) diagram which would have provided a useful tool to search for evidence of mode trapping. Considering Table 5-1 for $\ell=1$ modes, one finds only few modes of consecutive order separated by a number of missing modes. While the data are compatible with a distribution of periods covering as much as 23 orders, one finds 14 possibly consecutive order modes. The situation is similar for the $\ell=2$ modes (Table 5-2) where the entire period range covers 37 orders but where one finds only 9 possibly consecutive order modes. Hopefully, future observations may reveal additional modes which could fill the gaps so that a period spacingvs.-period diagram could eventually be built.

In comparing with the published models, those of Brassard et al. (1992) for instance, one finds a series close enough to HL Tau 76 with a total mass of $0.6 M_{\odot}$, effective temperature of $\approx 12000 \mathrm{~K}$, and a range in hydrogen mass fraction $-14 \leq$ $\log q_{\mathrm{H}} \leq-4$ and helium mass fraction $-8 \leq \log q_{\mathrm{He}} \leq-2$. The sensitivity of the period spacing to the various stellar parameters may be estimated from those results. For HL Tau 76, the total mass is $.55 \pm 0.03 M_{\odot}$ and $T_{\text {eff }}=11440 \pm 350 \mathrm{~K}$ (Bergeron et al. 1995). Within this small range of total mass and effective temperature, the dominant effect on the period spacing is due to the hydrogen mass fraction. In comparing with the various models of this sequence of Brassard's models (their Tables 2-5), one finds that HL Tau 76 period distribution is compatible with their moderately "thick" hydrogen models with $\log \left(q_{\mathrm{H}}\right) \geq-7.0$. A precise constraint on the stellar parameters of HL Tau 76 must await a detailed comparison of the periods calculated for realistic models of HL Tau 76 with the observations described here. This is out of the scope of the present paper and will be presented in a forthcoming publication.

\section{Summary}

We have analysed the light curve of HL Tau 76 obtained during the WET XCOV 18 campaign. We found as many as 78 peaks in the power spectrum. After eliminating the linear combinations, we are left with 36 significant independent modes. That is the richest spectrum found in any of the ZZ Ceti white dwarfs studied so far. The range of periods present in HL Tau 76 is exceptionally large, from $380 \mathrm{~s}$ for the shortest period to $1390 \mathrm{~s}$ for the longest. HL Tau 76 belongs to the group of ZZ Ceti stars close to the red edge of the instability strip which show both short and long periods. The presence of short periods in HL Tau 76, which belong to modes which seem to be still in the asymptotic regime, suggests that HL Tau 76 must have a moderatly "thick" hydrogen layer. We identified a few multiplets, doublets and triplets, which we interpret as $\ell=1$ modes split by rotation. From these multiplets we have derived an average period spacing of $48.0 \mathrm{~s}$ for the $\ell=1$ modes. By assuming that the modes observed in HL Tau 76 are in the asymptotic regime, we estimated the expected periods for consecutive or$\operatorname{der} \ell=1$ modes. The comparison with the observed periods leads to the identification of other $\ell=1$ modes. Some complex regions of the power spectrum are interpreted as due to overlapping $\ell=1$ and $\ell=2$ modes of close periods. We found some cases of doubtful identification since overlapping $\ell=1$ and $\ell=2$ modes are not rare. We also identify a number of $\ell=2$ modes which do not overlap with $\ell=1$ modes.

Having identified $\ell=1$ and $\ell=2$ modes in the XCOV18 data, we looked back in earlier data, the WET XCOV13 and earlier archival data, to check that the periods extracted from those data do fit satisfactorily the $\ell=1$ and $\ell=2$ identifications proposed here. In doing this, we found 7 more independent modes which fit nicely the expected period distribution. We propose a preliminary identification for most of the 43 modes detected in HL Tau 76. 
The rotation period derived from the rotational splitting is 2.2 days. There is no evidence for a dependence of the rotational splitting on the periods, which is compatible with the assumption that HL Tau 76 is rotating as a solid body at least in the outer layers.

Assuming the total mass and effective temperature well determined from spectroscopy, the period spacing may be used to estimate that the hydrogen mass fraction must be $\log \left(q_{\mathrm{H}}\right) \geq-7.0$. In spite of the large number of modes present in HL Tau 76, their distribution within an extended range, between $380 \mathrm{~s}$ and $1390 \mathrm{~s}$, does not allow to build a period spacing-vs.-Period diagram ( $\Delta P$-vs.- $P$ diagram) because of the large number of missing modes and the small number of consecutive order modes. As a consequence, it is not possible from the available data to derive information on potential mode trapping which would give another way to estimate the hydrogen mass fraction.

A detailed comparison between the observed periods determined in the present work and the periods of realistic models representative of HL Tau 76 should constrain more precisely the stellar parameters and the internal structure of HL Tau 76.

Acknowledgements. The WET acknowledges support from the U.S. National Science Foundation, through Grants AST-0205983 and AST-9877665 to Iowa State University. We thank Paul Bradley for his carefull reading of an earlier version of the manuscript and an anonymous referee whose recommendations helped improving a first version of this paper.

\section{References}

Abazajian, K., Adelman-McCarthy, J. K., Agüeros, M. A., et al. 2003, AJ, 126, 2081

Bergeron, P., Fontaine, G., Brassard, P., et al. 1993, AJ, 106, 1987

Bergeron, P., Wesemael, F., Lamontagne, R., et al. 1995, ApJ, 449, 258

Bergeron, P., Fontaine, G., Billères, M., Boudreault, S., \& Green, E. M. 2004, ApJ, 600, 404

Bradley, P. A. 1993, Ph.D. Thesis, University of Texas at Austin

Bradley, P. A. 1998, ApJS, 116, 307

Bradley, P. A. 2001, ApJ, 552, 326

Brassard, P., Fontaine, G., Wesemael, F., \& Tassoul, M. 1992, ApJS, 81,747

Brickhill, A. J. 1983, MNRAS, 204, 537

Brickhill, A. J. 1990, MNRAS, 246, 510

Brickhill, A. J. 1991a, MNRAS, 251, 537

Brickhill, A. J. 1991b, MNRAS, 252, 334

Clemens, J. C. 1993, Baltic Astron., 2, 407

Clemens, J. C., van Kerkwijk, M. H., \& Wu, Y. 2000, MNRAS, 314, 220

Corsico, A. H., Althaus, L. G., Benvenuto, O. G., \& Serenelli, A. M. 2002, A\&A, 387, 531

Dolez, N., \& Vauclair, G. 1981, A\&A, 102, 375

Dolez, N., \& Kleinman, S. J. 1997, in White Dwarfs, ed. I. Isern et al. (Kluwer Academic Publishers), 437

Fitch, W. S. 1973, ApJ, 181, L95

Fleming, T. A., Liebert, J., \& Green, R. F. 1986, ApJ, 308, 176

Fontaine, G., \& Brassard, P. 2004, 14th European Workshop on White Dwarfs, ed. D. Koester, \& S. Moehler, 565
Fontaine, G., Brassard, P., \& Bergeron, P. 2001, PASP, 113, 409

Fontaine, G., Bergeron, P., Billères, M., \& Charpinet, S. 2003, ApJ, 591,1184

Goldreich, P., \& Wu, Y. 1999a, ApJ, 511, 904

Goldreich, P., \& Wu, Y. 1999b, ApJ, 523, 805

Harris, H., Liebert, J., Kleinman, S. J., et al. 2003, AJ, 126, 1023

Jones, P. W., Hansen, C. J., Pesnell, W. D., et al. 1989, ApJ, 336, 403

Kanaan, A., Nitta, A., Winget, D. E., et al. 2005, A\&A, 432, 219

Kawaler, S. D., Sekii, T., \& Gough, D. 1999, ApJ, 516, 349

Kepler, S. O. 1993, BaltA, 2, 515

Kepler, S. O., Giovannini, O., Wood, M. A., et al. 1995, ApJ, 447, 874

Kepler, S. O., Mukadam, A., Winglet, D. E., et al. 2000a, ApJ, 534, 185

Kepler, S. O., Robinson, E. L., Koester, D., et al. 2000b, ApJ, 539, 379

Kepler, S. O., Nather, R. E., Winget, D. E., et al. 2003, A\&A, 401, 639

Kleinman, S. J., Nather, R. E., \& Phillips, T. 1996, PASP, 108, 356

Kleinman, S. J., Nather, R. E., Winget, D. E., et al. 1998, ApJ, 495, 424

Kleinman, S. J., Harris, H. C., Eisenstein, D. J., et al. 2004, ApJ, 607, 426

Kotak, R., van Kerkwijk, M. H., Clemens, J. C., \& Bida, T. A. 2002a, A\&A, 388, 219

Kotak, R., van Kerkwijk, M. H., Clemens, J. C., \& Bida, T. A. 2002b, A\&A, 391, 1005

Landolt, A. U. 1968, ApJ, 153, 151

Metcalfe, T. S., Montgomery, M. H., \& Kanaan, A. 2004, ApJ, 605, L133

Mukadam, A., Kepler, S. O., Winget, D. E., \& Bergeron, P. 2002, ApJ, 580,429

Mukadam, A., Mullaly, F., Nather, R. E., et al. 2004a, ApJ, 607, 982

Mukadam, A., Winget, D. E., von Hippel, T., et al. 2004b, ApJ, 612, 1052

Nather, R. E., Winget, D. E., Clemens, J. C., et al. 1990, ApJ, 361, 309

Page, C. G. 1972, MNRAS, 159, 25

Pfeiffer, B., Vauclair, G., Dolez, N., et al. 1996, A\&A, 314, 182

Robinson, E. L., Mailloux, T. M., Zhang, E., et al. 1995, ApJ, 438, 908

Scargle, J. D. 1982, ApJ, 263, 835

Tassoul, M. 1980, ApJS, 43, 469

Thompson, S., Clemens, J. C., van Kerkwijk, M. H., \& Koester, D. 2003, ApJ, 589, 1001

Thompson, S., Clemens, J. C., van Kerkwijk, M. H., et al. 2004, ApJ, 610,921

van Kerkwijk, M. H., Clemens, J. C., \& Wu. Y. 2000, MNRAS, 314, 209

Vauclair, G., Moskalik, P., Pfeiffer, B., et al. 2002, A\&A, 381, 122

Warner, B., \& Nather, R. E. 1970, MNRAS, 147, 21

Warner, B., \& Nather, R. E. 1972, MNRAS, 156, 1

Winget, D. E., \& Van Horn, H. M. 1987, IAU Coll. 95, The Second Conference on Faint Blue Stars, ed. A. G. Davis Philip, D. S. Hayes, \& J. W. Liebert (New York: L. Davis Press Inc., Schenectady), 363

Winget, D. E., Van Horn, H. M., Tassoul, M., et al. 1982, ApJ, 252, L65

Winget, D. E., Hansen, C. J., Liebert, J. W., et al. 1987, ApJ, 315, 77

Winget, D. E., Nather, R. E., Clemens, J. C., et al. 1991, ApJ, 378, 326

Winget, D. E., Nather, R. E., Clemens, J. C., et al. 1994, ApJ, 430, 839

Wood, M. A. 1995, in White Dwarfs, ed. D. Koester, \& K. Werner (Berlin: Springer), 41

Wu, Y. 2001, MNRAS, 323, 248

Wu, Y., \& Goldreich, P. 1999, ApJ, 519, 783

Wu, Y., \& Goldreich, P. 2001, ApJ, 546, 469 
1 Université Paul Sabatier, Observatoire Midi-Pyrénées, CNRS/UMR5572, 14 Av. E. Belin, 31400 Toulouse, France

2 Sloan Digital Sky Survey, Apache Point Observatory, PO Box 59, Sunspot, NM 88349, USA

3 Observatoire de Paris-Meudon, LESIA, 92195 Meudon, France

4 Department of Physics, University of Troms $\varnothing$, 9037 Troms $\varnothing$, Norway

5 Departamento de Fisica Aplicada, Universidade de Vigo, 36200 Vigo, Spain

${ }^{6}$ Universidade Federal de Santa Catarina, Florianopolis, SC, Brazil

7 Department of Physics and Astronomy, Iowa State University, Ames, IA 50011, USA

8 Department of Astronomy and McDonald Observatory, Texas University at Austin, Austin, TX 78712, USA

${ }^{9}$ Department of Physics and Space Sciences and SARA Observatory, Florida Institute of Technology, Melbourne, FL 32901, USA

10 School of Chemical and Physical Sciences, Victoria University of Wellington, PO Box 600, Wellington, New-Zealand

11 National Astronomical Observatories, Chinese Academy of Sciences, 20A, Datun Road, Beijing 100012, PR China

12 Indian Space Research Organization, Airport Road, Vimanapura PO, Bangalore 560 017, India

13 Wise Observatory, Tel Aviv University, Tel Aviv 69978, Israel

14 South African Astronomical Observatory, PO Box 9, Cape Town, 7935 South-Africa
15 Vilnius University Research Institute of Theoretical Physics and Astronomy, A. Gostauto 12, 01108 Vilnius, Lithuania

16 Copernicus Astronomical Center, Ul. Bartycka 18, 00-716 Warsaw, Poland

17 Mt. Suhora Observatory, Cracow Pedagogical University, Ul. Podchorazych 2, 30-084 Cracow, Poland

18 Institut für Astronomie Universität Wien, Tükenschanzstrasse 17, 1180, Austria

19 Osservatorio Astronomico di Capodimonte, via Moiariello 16, 80131, Napoli, Italy

20 Astronomy Group, Vrije Universiteit Brussel (VUB), Pleinlaan 2, 1050 Brussel, Belgium

${ }^{21}$ Institute of Theoretical Astrophysics, University of Oslo, P. Box 1029, 0315 Oslo, Norway

22 Present address: Instituto de Astrofisica de Canarias, Tenerife, Spain

23 Present address: Department of Physics, Astronomy, and Material Science, SW Missouri State University, Springfield, MO 65804, USA

24 Present address: High Altitude Observatory, PO Box 3000, Boulder, CO 80307-3000, USA

25 Present address: Department of Physics and Astronomy, Middle Tennessee State University, Murfreesboro, TN 37132, USA

26 Present address Department of Astronomy, Box 351580, University of Washington, Seattle, WA 98195-1580, USA 


\section{Online Material}


N. Dolez et al.: Asteroseismology of HL TAU 76, Online Material p 2

Table 2. Journal of observations: 1999 WET (XCOV18).

\begin{tabular}{|c|c|c|c|c|}
\hline Run name & Telescope & $\begin{array}{l}\text { Date } \\
\text { (UT) }\end{array}$ & $\begin{array}{c}\text { Start time } \\
\text { (UTC) }\end{array}$ & Run length (s) \\
\hline suh-79 & Suhora $0.6 \mathrm{~m}$ & 30 Oct. 99 & $20: 52: 00$ & 15440 \\
\hline jxj-9935 & $\mathrm{BAO} 0.85 \mathrm{~m}$ & 1 Nov. 99 & $13: 36: 40$ & 28480 \\
\hline suh- 80 & Suhora $0.6 \mathrm{~m}$ & 1 Nov. 99 & $18: 24: 20$ & 32240 \\
\hline tsm-0050 & McDonald $2.1 \mathrm{~m}$ & 2 Nov. 99 & 04:39:30 & 21810 \\
\hline sara-016 & KPNO SARA $0.9 \mathrm{~m}$ & 2 Nov. 99 & 05:11:08 & $19010 \mathrm{CCD}$ \\
\hline jxj-9937 & $\mathrm{BAO} 0.85 \mathrm{~m}$ & 2 Nov. 99 & 13:53:00 & 28030 \\
\hline suh-81 & Suhora $0.6 \mathrm{~m}$ & 2 Nov. 99 & $17: 48: 30$ & 32800 \\
\hline teide01 & IAC $0.8 \mathrm{~m}$ & 3 Nov. 99 & $02: 10: 30$ & 14920 \\
\hline sara-017 & KPNO SARA $0.9 \mathrm{~m}$ & 3 Nov. 99 & 04:09:35 & $6210 \mathrm{CCD}$ \\
\hline tsm-0052 & McDonald $2.1 \mathrm{~m}$ & 3 Nov. 99 & 04:40:00 & 26760 \\
\hline jxj-9938 & BAO $0.85 \mathrm{~m}$ & 3 Nov. 99 & $13: 41: 00$ & 28780 \\
\hline teide02 & IAC $0.8 \mathrm{~m}$ & 3 Nov. 99 & $23: 45: 50$ & 23830 \\
\hline sara-018 & KPNO SARA $0.9 \mathrm{~m}$ & 4 Nov. 99 & 03:51:13 & $32700 \mathrm{CCD}$ \\
\hline jxj-9939 & $\mathrm{BAO} 0.85 \mathrm{~m}$ & 4 Nov. 99 & $13: 23: 20$ & 30010 \\
\hline suh-82 & Suhora $0.6 \mathrm{~m}$ & 4 Nov. 99 & 17:21:00 & 34900 \\
\hline HL1104OI & OHP $1.9 \mathrm{~m}$ & 4 Nov. 99 & $21: 45: 00$ & 24240 \\
\hline tsm-0054 & McDonald $2.1 \mathrm{~m}$ & 5 Nov. 99 & $04: 21: 30$ & 28110 \\
\hline sara-019 & KPNO SARA $0.9 \mathrm{~m}$ & 5 Nov. 99 & $05: 16: 28$ & $28280 \mathrm{CCD}$ \\
\hline suh- 83 & Suhora $0.6 \mathrm{~m}$ & 5 Nov. 99 & $17: 57: 00$ & 31850 \\
\hline sara- 020 & KPNO SARA $0.9 \mathrm{~m}$ & 6 Nov. 99 & $03: 12: 32$ & 9910 CCD \\
\hline tsm-0056 & McDonald $2.1 \mathrm{~m}$ & 6 Nov. 99 & $04: 42: 30$ & 26250 \\
\hline N49-0423 & Naini Tal $1.0 \mathrm{~m}$ & 6 Nov. 99 & 19:51:30 & 14060 \\
\hline HL1106OJ & OHP $1.9 \mathrm{~m}$ & 6 Nov. 99 & 22:01:00 & 22820 \\
\hline tsm-0058 & McDonald $2.1 \mathrm{~m}$ & 7 Nov. 99 & $04: 17: 30$ & 28650 \\
\hline jxj-9940 & $\mathrm{BAO} 0.85 \mathrm{~m}$ & 7 Nov. 99 & $13: 30: 30$ & 30700 \\
\hline n49-0424 & Naini Tal $1.0 \mathrm{~m}$ & 7 Nov. 99 & $17: 25: 00$ & 23010 \\
\hline hl1107oi & OHP $1.9 \mathrm{~m}$ & 7 Nov. 99 & 20:09:00 & 27040 \\
\hline teide04 & IAC $0.8 \mathrm{~m}$ & 8 Nov. 99 & 01:30:10 & 17750 \\
\hline mdr073 & CTIO $1.5 \mathrm{~m}$ & 8 Nov. 99 & 04:39:10 & 14570 \\
\hline no0899q1 & Hawaii UH 0.6 m & 8 Nov. 99 & 08:48:40 & 24110 \\
\hline jxj-9941 & BAO $0.85 \mathrm{~m}$ & 8 Nov. 99 & $13: 15: 30$ & 30490 \\
\hline wccd-001 & Wise, $1 \mathrm{~m}$ & 8 Nov. 99 & 18:30:00 & $27580 \mathrm{CCD}$ \\
\hline HL1108OJ & OHP $1.93 \mathrm{~m}$ & 8 Nov. 99 & 20:53:00 & 27160 \\
\hline teide 05 & IAC $0.8 \mathrm{~m}$ & 9 Nov. 99 & 00:06:30 & 22360 \\
\hline sara-021 & KPNO SARA $0.9 \mathrm{~m}$ & 9 Nov. 99 & $03: 38: 30$ & $32200 \mathrm{CCD}$ \\
\hline mdr076 & CTIO $1.5 \mathrm{~m}$ & 9 Nov. 99 & $04: 37: 50$ & 6130 \\
\hline
\end{tabular}


Table 2. continued.

\begin{tabular}{|c|c|c|c|c|}
\hline Run name & Telescope & $\begin{array}{l}\text { Date } \\
\text { (UT) }\end{array}$ & $\begin{array}{c}\text { Start time } \\
\text { (UTC) }\end{array}$ & Run length (s) \\
\hline no0999q1 & Hawaii $0.6 \mathrm{~m}$ & 9 Nov. 99 & 07:54:40 & 27440 \\
\hline tsm-0059 & McDonald $2.1 \mathrm{~m}$ & 9 Nov. 99 & 09:04:00 & 11160 \\
\hline wccd-005 & Wise $1.0 \mathrm{~m}$ & 9 Nov. 99 & 18:57:00 & $16990 \mathrm{CCD}$ \\
\hline HL1109OI & OHP $1.93 \mathrm{~m}$ & 9 Nov. 99 & 21:11:00 & 28230 \\
\hline teide 06 & IAC $0.8 \mathrm{~m}$ & 9 Nov. 99 & $21: 26: 30$ & 26500 \\
\hline lo09-1.dat & Loiano $1.5 \mathrm{~m}$ & 9 Nov. 99 & $22: 32: 40$ & 20660 \\
\hline sara-022 & KPNO SARA $0.9 \mathrm{~m}$ & 10 Nov. 99 & 03:05:29 & $14100 \mathrm{CCD}$ \\
\hline tsm-0061 & McDonald $2.1 \mathrm{~m}$ & 10 Nov. 99 & $03: 25: 30$ & 30600 \\
\hline no1099q1 & Hawaii $0.6 \mathrm{~m}$ & 10 Nov. 99 & 07:54:00 & 27350 \\
\hline N49-0429 & Naini Tal $1 \mathrm{~m}$ & 10 Nov. 99 & $17: 52: 30$ & 21470 \\
\hline wccd-008 & Wise $1.0 \mathrm{~m}$ & 10 Nov. 99 & $18: 49: 10$ & $27970 \mathrm{CCD}$ \\
\hline HL1110OJ & OHP $1.9 \mathrm{~m}$ & 10 Nov. 99 & $20: 35: 00$ & 29470 \\
\hline teide 07 & IAC $0.8 \mathrm{~m}$ & 10 Nov. 99 & $23: 12: 30$ & 25260 \\
\hline tsm-0063 & McDonald $2.1 \mathrm{~m}$ & 11 Nov. 99 & 03:20:00 & 32400 \\
\hline no1199q3 & Hawaii $0.6 \mathrm{~m}$ & 11 Nov. 99 & $11: 26: 40$ & 14690 \\
\hline jxj-9942 & $\mathrm{BAO} 0.85 \mathrm{~m}$ & 11 Nov. 99 & $17: 23: 20$ & 2140 \\
\hline wccd-013 & Wise $1.0 \mathrm{~m}$ & 11 Nov. 99 & $18: 54: 20$ & $28500 \mathrm{CCD}$ \\
\hline teide 08 & IAC $0.8 \mathrm{~m}$ & 11 Nov. 99 & $21: 12: 30$ & 29850 \\
\hline tsm-0066 & McDonald $2.1 \mathrm{~m}$ & 12 Nov. 99 & $03: 25: 30$ & 20200 \\
\hline no1299q1 & Hawaii $0.6 \mathrm{~m}$ & 12 Nov. 99 & 07:20:10 & 28140 \\
\hline jxj-9943 & BAO $0.85 \mathrm{~m}$ & 12 Nov. 99 & $13: 12: 20$ & 11920 \\
\hline suh-84 & Suhora $0.6 \mathrm{~m}$ & 12 Nov. 99 & 17:08:10 & 40000 \\
\hline mdr085 & CTIO $1.5 \mathrm{~m}$ & 13 Nov. 99 & 04:29:20 & 13850 \\
\hline no1399q1 & Hawaii $0.6 \mathrm{~m}$ & 13 Nov. 99 & 07:09:30 & 24390 \\
\hline tsm-0067 & McDonald $2.1 \mathrm{~m}$ & 13 Nov. 99 & 07:29:30 & 16830 \\
\hline suh-86 & Suhora $0.6 \mathrm{~m}$ & 13 Nov. 99 & 17:04:10 & 19720 \\
\hline tsm-0069 & McDonald $2.1 \mathrm{~m}$ & 14 Nov. 99 & 03:13:30 & 31100 \\
\hline no1499q2 & Hawaii $0.6 \mathrm{~m}$ & 14 Nov. 99 & $11: 31: 10$ & 14450 \\
\hline edrim 01 & Moletai $1.65 \mathrm{~m}$ & 14 Nov. 99 & $19: 18: 20$ & 4450 \\
\hline edrim 02 & Moletai $1.65 \mathrm{~m}$ & 15 Nov. 99 & $01: 23: 20$ & 3510 \\
\hline tsm-0070 & McDonald $2.1 \mathrm{~m}$ & 15 Nov. 99 & 02:53:00 & 32820 \\
\hline no1599q3 & Hawaii $0.6 \mathrm{~m}$ & 15 Nov. 99 & 11:44:50 & 13340 \\
\hline suh-87 & Suhora $0.6 \mathrm{~m}$ & 15 Nov. 99 & 21:00:10 & 27230 \\
\hline tsm-0072 & McDonald $2.1 \mathrm{~m}$ & 16 Nov. 99 & 03:43:00 & 29910 \\
\hline no1699q2 & Hawaii $0.6 \mathrm{~m}$ & 16 Nov. 99 & 11:20:00 & 14700 \\
\hline suh- 88 & Suhora $0.6 \mathrm{~m}$ & 16 Nov. 99 & $16: 40: 00$ & 17620 \\
\hline no1799q1 & Hawaii $0.6 \mathrm{~m}$ & 17 Nov. 99 & 09:43:00 & 19810 \\
\hline
\end{tabular}

